\title{
AN EFFICIENT GREEN PROTOCOL FOR THE SYNTHESIS OF NOVEL DIHYDROINDENO[1,2-b]PYRROLES
}

\author{
Veerappan Jeyachandran, Muthumani Muthu and Raju Ranjith Kumar* \\ Department of Organic Chemistry, School of Chemistry, Madurai Kamaraj University, \\ Madurai - 625021, Tamil Nadu, India
}

\section{SUPPLEMENTARY INFORMATION}

\section{EXPERIMENTAL}

\section{General}

The melting points were measured in open capillary tubes and are uncorrected. The

${ }^{1} \mathrm{H},{ }^{13} \mathrm{C}$ and the 2D NMR spectra were recorded on a Bruker (Avance) $300 \mathrm{MHz}$ NMR instrument using TMS as internal standard and $\mathrm{CDCl}_{3}$ as solvent. Standard Bruker software was used throughout. Chemical shifts are given in parts per million ( $\delta$-scale) and the coupling constants are given in Hertz. Elemental analyses were performed on a Perkin Elmer 2400 Series II Elemental CHNS analyzer. All the chemicals were purchased from Sigma-Aldrich and used without any further purification. Single crystal X-ray studies of $\mathbf{5 a}, \mathbf{5 d}$ and $\mathbf{5 h}$, Data collection: APEX2 (Bruker, 2004); cell refinement: SAINT (Bruker, 2004); data reduction: SAINT (Bruker, 2004); program(s) used to solve structure: SHELXS97 (Sheldrick, 2008); program(s) used to refine structure: SHELXL97 (Sheldrick, 2008); molecular graphics: PLATON (Spek, 2009); software used: SHELXL97 (Sheldrick, 2008).

General procedure for the synthesis of dihydroindeno[1,2-b]pyrrol-4(3aH)-ones 4 and 5 
An equimolar mixture of (E)- $N$-methyl-1-(methylthio)-2-nitroethenamine 1 or $(E)-N$ (1-(methylthio)-2-nitrovinyl)anilines 2 and ninhydrin 3 were dissolved in ethanol $(2-3 \mathrm{~mL})$ and stirred at ambient temperature for $5 \mathrm{~min}$. Completion of the reaction was noted when the product precipitated from the reaction mixture, which was a clear solution at the beginning of the reaction. The precipitate was filtered, washed with water $(10 \mathrm{~mL})$ and dried to obtain novel 3a,8b-dihydroxy-1-methyl-2-(methylthio)-3-nitro-1,8b-dihydroindeno[1,2-b]pyrrol4(3aH)-one 4 or 3a,8b-dihydroxy-2-(methylthio)-3-nitro-1-phenyl-1,8b-di-hydroindeno[1,2b]pyrrol-4(3aH)-ones 5 in quantitative yields.

\section{3a,8b-Dihydroxy-1-methyl-2-(methylthio)-3-nitro-1,8b-dihydroindeno[1,2-b]pyrrol-}

\section{4(3aH)-one (4)}

Isolated as pale yellow solid; Yield 99 \%; m.p. $195-197{ }^{\circ} \mathrm{C} ;{ }^{1} \mathrm{H}$ NMR (300 MHz, $\left.\mathrm{CDCl}_{3}\right)$ $\delta_{\mathrm{H}}: 2.48(\mathrm{~s}, 3 \mathrm{H}), 3.36(\mathrm{~s}, 3 \mathrm{H}), 6.03(\mathrm{~s}, 1 \mathrm{H}), 6.49(\mathrm{~s}, 1 \mathrm{H}), 7.47-7.52(\mathrm{~m}, 1 \mathrm{H}), 7.66-7.68(\mathrm{~m}$,

2H), 7.47-7.52 (m, 1H), $7.76(\mathrm{~d}, J=7.5 \mathrm{~Hz}, 1 \mathrm{H}) ; \mathrm{C}^{13} \mathrm{NMR}\left(75 \mathrm{MHz}, \mathrm{CDCl}_{3}\right) \delta_{\mathrm{C}}: 17.0,29.6$, 81.1, 94.8, 120.6, 123.6, 123.8, 130.4, 134.2, 135.5, 145.8, 161.1 and 194.8. Anal. Calcd. for $\mathrm{C}_{13} \mathrm{H}_{12} \mathrm{~N}_{2} \mathrm{O}_{5} \mathrm{~S}:$ C, 50.65; H, 3.92; N, 9.09\%. Found: C, 50.63; H, 3.88; N, 9.04\%.

3a,8b-Dihydroxy-2-(methylthio)-3-nitro-1-phenyl-1,8b-dihydroindeno[1,2-b]pyrrol4(3aH)-one (5a)

Isolated as pale yellow solid; Yield 94\%; m.p. $178-180{ }^{\circ} \mathrm{C} ;{ }^{1} \mathrm{H}$ NMR $\left(300 \mathrm{MHz}, \mathrm{CDCl}_{3}\right) \delta_{\mathrm{H}}$ : $1.87(\mathrm{~s}, 3 \mathrm{H}), 6.58(\mathrm{~d}, J=7.2 \mathrm{~Hz}, 1 \mathrm{H}), 7.42-7.55(\mathrm{~m}, 6 \mathrm{H}), 7.87(\mathrm{~d}, J=7.8 \mathrm{~Hz}, 1 \mathrm{H}) ; \mathrm{C}^{13} \mathrm{NMR}$ $\left(75 \mathrm{MHz}, \mathrm{CDCl}_{3}\right) \delta_{\mathrm{C}}: 16.8,80.6,97.6,122.3,124.6,125.5,129.3,129.4,129.7,131.1,134.5$, 135.6, 136.7, 145.9, 163.8 and 195.1. Anal. Calcd. for $\mathrm{C}_{19} \mathrm{H}_{16} \mathrm{~N}_{2} \mathrm{O}_{5} \mathrm{~S}: \mathrm{C}, 58.37 ; \mathrm{H}, 3.81$; , 7.56\%. Found: C, 58.35; H, 3.83; N, 7.54\%.

3a,8b-Dihydroxy-2-(methylthio)-3-nitro-1-o-tolyl-1,8b-dihydroindeno[1,2-b]pyrrol4(3aH)-one (5b) 
Isolated as pale yellow solid; Yield 96\%; m.p. $200-202{ }^{\circ} \mathrm{C}:{ }^{1} \mathrm{H} \mathrm{NMR}\left(300 \mathrm{MHz}, \mathrm{CDCl}_{3}\right) \delta_{\mathrm{H}}$ : $1.34(\mathrm{~s}, 3 \mathrm{H}), 1.87(\mathrm{~s}, 3 \mathrm{H}), 4.94(\mathrm{~s}, 1 \mathrm{H}), 5.97(\mathrm{~s}, 1 \mathrm{H}), 6.49$ (d, $J=7.8 \mathrm{~Hz}, 1 \mathrm{H}), 7.12-7.17(\mathrm{~s}$, 1H), 7.39-7.58 (m, 4H), 7.77-7.80 ( m, 1H), $7.89(\mathrm{~d}, J=7.5 \mathrm{~Hz}, 1 \mathrm{H}) ; \mathrm{C}^{13} \mathrm{NMR}(75 \mathrm{MHz}$, $\left.\mathrm{CDCl}_{3}\right) \delta_{\mathrm{C}}: 16.0,17.3,80.7,97.7,1205,124.4,125.1,127.2,129.9,130.9,131.9,134.1$, 134.9, 135.3, 138.2, 146.3, 163.0 and 195.7. Anal. Calcd. for $\mathrm{C}_{19} \mathrm{H}_{16} \mathrm{~N}_{2} \mathrm{O}_{5} \mathrm{~S}: \mathrm{C}, 59.37 ; \mathrm{H}$, 4.20; N, 7.29\%. Found: C, 59.35; H, 4.17; N, 7.26\%.

3a,8b-Dihydroxy-2-(methylthio)-3-nitro-1-m-tolyl-1,8b-dihydroindeno[1,2-b]pyrrol4(3aH)-one (5c)

Isolated as pale yellow solid; Yield 96\%; m.p. $195-197{ }^{\circ} \mathrm{C} ;{ }^{1} \mathrm{H}$ NMR $\left(300 \mathrm{MHz}, \mathrm{CDCl}_{3}\right) \delta_{\mathrm{H}}$ : $1.69(\mathrm{~s}, 3 \mathrm{H}), 1.88(\mathrm{~s}, 3 \mathrm{H}), 4.82(\mathrm{~s}, 1 \mathrm{H}), 5.99(\mathrm{~s}, 1 \mathrm{H}), 6.61(\mathrm{~d}, J=7.8 \mathrm{~Hz}, 1 \mathrm{H}), 7.31-7.56(\mathrm{~m}$,

$6 \mathrm{H}), 7.88(\mathrm{~d}, J=7.5 \mathrm{~Hz}, 1 \mathrm{H}) ; \mathrm{C}^{13} \mathrm{NMR}\left(75 \mathrm{MHz}, \mathrm{CDCl}_{3}\right) \delta_{\mathrm{C}}: 16.7,21.2,80.4,97.1,122.2$, $124.6,125.4,126.1,129.4,129.5,130.1,131.1,134.5,135.4,136.4,139.9,145.9,163.5$ and 194.7. Anal. Calcd. for $\mathrm{C}_{19} \mathrm{H}_{16} \mathrm{~N}_{2} \mathrm{O}_{5} \mathrm{~S}: \mathrm{C}, 59.37 ; \mathrm{H}, 4.20 ; \mathrm{N}, 7.29 \%$. Found: C, 59.33; H, 4.17; N, $7.27 \%$.

3a,8b-Dihydroxy-2-(methylthio)-3-nitro-1-p-tolyl-1,8b-dihydroindeno[1,2-b]pyrrol4(3aH)-one (5d)

Isolated as pale yellow solid; Yield 96\%; m.p. $199-201{ }^{\circ} \mathrm{C} ;{ }^{1} \mathrm{H}$ NMR $\left(300 \mathrm{MHz}, \mathrm{CDCl}_{3}\right) \delta_{\mathrm{H}}$ : $1.87(\mathrm{~s}, 3 \mathrm{H}), 2.11(\mathrm{~s}, 3 \mathrm{H}), 6.57(\mathrm{~d}, J=7.8 \mathrm{~Hz}, 1 \mathrm{H}), 7.43-7.56(\mathrm{~m}, 6 \mathrm{H}), 7.89(\mathrm{~d}, J=7.5 \mathrm{~Hz}$, $1 \mathrm{H}) ; \mathrm{C}^{13} \mathrm{NMR}\left(75 \mathrm{MHz}, \mathrm{CDCl}_{3}\right) \delta_{\mathrm{C}}: 16.7,20.6,80.5,97.2,122.4,124.6,125.4,129.2$, $129.3,129.6,131.1,134.5,135.5,136.7,146.0,163.4,176.5$ and 195.0. Anal. Calcd. for $\mathrm{C}_{19} \mathrm{H}_{16} \mathrm{~N}_{2} \mathrm{O}_{5} \mathrm{~S}: \mathrm{C}, 59.37 ; \mathrm{H}, 4.20 ; \mathrm{N}, 7.29 \%$. Found: C, 59.33; H, 4.18; N, 7.26\%.

1-(2,4-Dimethylphenyl)-3a,8b-dihydroxy-2-(methylthio)-3-nitro-1,8bdihydroindeno[1,2-b] pyrrol-4(3aH)-one (5e)

Isolated as pale yellow solid; Yield 96\%; m.p. $202-204{ }^{\circ} \mathrm{C} ;{ }^{1} \mathrm{H}$ NMR $\left(300 \mathrm{MHz}, \mathrm{CDCl}_{3}\right) \delta_{\mathrm{H}}$ : $1.29(\mathrm{~s}, 3 \mathrm{H}), 1.89(\mathrm{~s}, 3 \mathrm{H}), 2.42(\mathrm{~s}, 3 \mathrm{H}), 4.93(\mathrm{~s}, 1 \mathrm{H}), 5.31(\mathrm{~s}, 1 \mathrm{H}), 6.55(\mathrm{~d}, J=7.5 \mathrm{~Hz}, 1 \mathrm{H})$, 
$7.23(\mathrm{~d}, J=8.4 \mathrm{~Hz}, 1 \mathrm{H}), 7.45(\mathrm{t}, J=7.8 \mathrm{~Hz}, 1 \mathrm{H}), 7.55(\mathrm{t}, J=7.5 \mathrm{~Hz}, 1 \mathrm{H}), 7.65(\mathrm{~d}, J=8.4 \mathrm{~Hz}$, 1H), $7.89(\mathrm{~d}, J=7.8 \mathrm{~Hz}, 1 \mathrm{H}) ; \mathrm{C}^{13} \mathrm{NMR}\left(75 \mathrm{MHz}, \mathrm{CDCl}_{3}\right) \delta_{\mathrm{C}}: 16.5,17.3,21.3,79.3,97.5$, 124.7, 125.1, 128.1, 130.6, 131.0, 134.8, 135.5, 137.8, 140.4, 146.0 and 194.9. Anal. Calcd. for $\mathrm{C}_{20} \mathrm{H}_{18} \mathrm{~N}_{2} \mathrm{O}_{5} \mathrm{~S}$ : C, 60.29; H, 4.55; N, 7.03\%. Found: C, 60.27; H, 4.51; N, 7.01\%.

1-(2,5-Dimethylphenyl)-3a,8b-dihydroxy-2-(methylthio)-3-nitro-1,8bdihydroindeno[1,2-b] pyrrol-4(3aH)-one (5f)

Isolated as pale yellow solid; Yield 95\%; m.p. $215-217{ }^{\circ} \mathrm{C} ;{ }^{1} \mathrm{H}$ NMR $\left(300 \mathrm{MHz}, \mathrm{CDCl}_{3}\right) \delta_{\mathrm{H}}$ : $1.28(\mathrm{~s}, 3 \mathrm{H}), 1.89(\mathrm{~s}, 3 \mathrm{H}), 2.46(\mathrm{~s}, 3 \mathrm{H}), 4.92(\mathrm{~s}, 1 \mathrm{H}), 5.97(\mathrm{~s}, 1 \mathrm{H}), 6.53(\mathrm{~d}, J=7.5 \mathrm{~Hz}, 1 \mathrm{H})$, $7.01(\mathrm{~d}, J=8.1 \mathrm{~Hz}, 1 \mathrm{H}), 7.23-7.29(\mathrm{~m}, 1 \mathrm{H}), 7.43(\mathrm{t}, J=7.8 \mathrm{~Hz}), 7.51-7.58(\mathrm{~m}, 1 \mathrm{H}), 7.89(\mathrm{~d}$, $J=7.2 \mathrm{~Hz}, 1 \mathrm{H}) ; \mathrm{C}^{13} \mathrm{NMR}\left(75 \mathrm{MHz}, \mathrm{CDCl}_{3}\right) \delta_{\mathrm{C}}: 21.2,21.6,84.8,102.8,128.9,130.1,135.1$, 135.6, 135.7, 136.2, 138.6, 139.7, 140.0, 141.2, 141.6, 151.1, 167.9 and 200.6. Anal. Calcd. for $\mathrm{C}_{19} \mathrm{H}_{16} \mathrm{~N}_{2} \mathrm{O}_{6} \mathrm{~S}: \mathrm{C}, 60.29 ; \mathrm{H}, 4.55 ; \mathrm{N}, 7.03 \%$. Found: C, 60.27; H, 4.54; N, 7.01\%.

3a,8b-Dihydroxy-1-(2-methoxyphenyl)-2-(methylthio)-3-nitro-1,8b-dihydroindeno[1,2-

\section{b] pyrrol-4(3aH)-one (5g)}

Isolated as pale yellow solid; Yield 94\%; m.p. $209-211{ }^{\circ} \mathrm{C} ;{ }^{1} \mathrm{H} \mathrm{NMR}\left(300 \mathrm{MHz}, \mathrm{CDCl}_{3}\right) \delta_{\mathrm{H}}$ : $1.98(\mathrm{~s}, 3 \mathrm{H}), 2.22(\mathrm{~s}, 3 \mathrm{H}), 4.78(\mathrm{~s}, 1 \mathrm{H}), 5.77(\mathrm{~s}, 1 \mathrm{H}), 6.57(\mathrm{~d}, J=7.8 \mathrm{~Hz}, 1 \mathrm{H}), 6.80(\mathrm{~d}, J=$ $8.4 \mathrm{~Hz}, 1 \mathrm{H}), 7.16(\mathrm{t}, J=7.8 \mathrm{~Hz}, 1 \mathrm{H}), 7.40(\mathrm{t}, J=7.5 \mathrm{~Hz}, 1 \mathrm{H}), 7.37-7.53(\mathrm{~m}, 2 \mathrm{H}), 7.69$ (dd, $J=$ $1.5 \mathrm{~Hz}, J=7.8 \mathrm{~Hz}, 1 \mathrm{H}), 7.87(\mathrm{~d}, J=7.2 \mathrm{~Hz}, 1 \mathrm{H}) ; \mathrm{C}^{13} \mathrm{NMR}\left(75 \mathrm{MHz}, \mathrm{CDCl}_{3}\right) \delta_{\mathrm{C}}: 15.8,54.6$, $79.9,96.9,110.6,120.4,120.9,123.2,124.0,124.4,129.9,130.7,134.2,146.4,155.7,164.1$ and 195.5. Anal. Calcd. for $\mathrm{C}_{19} \mathrm{H}_{16} \mathrm{~N}_{2} \mathrm{O}_{6} \mathrm{~S}: \mathrm{C}, 56.99 ; \mathrm{H}, 4.03 ; \mathrm{N}, 7.00 \%$. Found: 56.95; H, $4.00 ; \mathrm{N}, 6.99 \%$.

3a,8b-Dihydroxy-1-(4-methoxyphenyl)-2-(methylthio)-3-nitro-1,8b-dihydroindeno[1,2b] pyrrol-4(3aH)-one (5h)

Isolated as pale yellow solid; Yield 95\%; m.p. $200-202{ }^{\circ} \mathrm{C} ;{ }^{1} \mathrm{H} \mathrm{NMR}\left(300 \mathrm{MHz}, \mathrm{CDCl}_{3}\right) \delta_{\mathrm{H}}$ : $1.91(\mathrm{~s}, 3 \mathrm{H}), 3.91(\mathrm{~s}, 3 \mathrm{H}), 4.82(\mathrm{~s}, 1 \mathrm{H}), 5.56(\mathrm{~s}, 1 \mathrm{H}), 6.64(\mathrm{~d}, J=7.5 \mathrm{~Hz}, 1 \mathrm{H}), 6.97-7.0(\mathrm{~m}$, 
$3 \mathrm{H}), 7.45-7.56(\mathrm{~m}, 3 \mathrm{H}), 7.88(\mathrm{~d}, J=7.5 \mathrm{~Hz}, 1 \mathrm{H}) ; \mathrm{C}^{13} \mathrm{NMR}\left(75 \mathrm{MHz}, \mathrm{CDCl}_{3}\right) \delta_{\mathrm{C}}: 16.7,55.5$, $80.8,97.8,114.5,122.3,124.2,125.6,128.8,130.6,130.8,134.7,135.2,146.3,159.9,163.2$ and 195.4. Anal. Calcd. for $\mathrm{C}_{19} \mathrm{H}_{16} \mathrm{~N}_{2} \mathrm{O}_{6} \mathrm{~S}: \mathrm{C}, 56.99 ; \mathrm{H}, 4.03 ; \mathrm{N}, 7.00 \%$. Found: 56.93; H, $4.01 ; \mathrm{N}, 6.96 \%$.

1-(4-Chlorophenyl)-3a,8b-dihydroxy-2-(methylthio)-3-nitro-1,8b-dihydroindeno[1,2-b] pyrrol-4(3aH)-one (5i)

Isolated as pale yellow solid; Yield 92\%; m.p. $187-189^{\circ} \mathrm{C} ;{ }^{1} \mathrm{H}$ NMR $\left(300 \mathrm{MHz}, \mathrm{CDCl}_{3}\right) \delta_{\mathrm{H}}$ : $1.95(\mathrm{~s}, 3 \mathrm{H}), 5.00(\mathrm{~s}, 1 \mathrm{H}), 5.57(\mathrm{~s}, 1 \mathrm{H}), 6.67(\mathrm{~d}, J=7.5 \mathrm{~Hz}, 1 \mathrm{H}), 7.37-7.59(\mathrm{~m}, 6 \mathrm{H}), 7.89$ (d, $J$ $=7.5 \mathrm{~Hz}, 1 \mathrm{H}) ; \mathrm{C}^{13} \mathrm{NMR}\left(75 \mathrm{MHz}, \mathrm{CDCl}_{3}\right) \delta_{\mathrm{C}}: 16.9,80.6,97.4,122.6,124.7,125.2,129.8$, 130.6, 131.2, 134.5, 135.2, 135.3, 135.7, 145.7, 163.2 and 194.7. Anal. Calcd. for $\mathrm{C}_{18} \mathrm{H}_{13} \mathrm{ClN}_{2} \mathrm{O}_{5} \mathrm{~S}: \mathrm{C}, 53.41 ; \mathrm{H}, 3.24 ; \mathrm{N}, 6.92 \%$. Found: C, 53.37; H, 3.21; N, 6.90\%.

\section{1-(4-Fluorophenyl)-3a,8b-dihydroxy-2-(methylthio)-3-nitro-1,8b-dihydroindeno[1,2-b]}

\section{pyrrol-4(3aH)-one (5j)}

Isolated as pale yellow solid; Yield 93\%; m.p. $182-184{ }^{\circ} \mathrm{C} ;{ }^{1} \mathrm{H}$ NMR $\left(300 \mathrm{MHz}, \mathrm{CDCl}_{3}\right) \delta_{\mathrm{H}}$ : $1.94(\mathrm{~s}, 3 \mathrm{H}), 6.62(\mathrm{~d}, J=7.8 \mathrm{~Hz}, 1 \mathrm{H}), 7.16-7.25(\mathrm{~m}, 3 \mathrm{H}), 7.48-7.59(\mathrm{~m}, 3 \mathrm{H}), 7.88(\mathrm{~d}, J=$

$7.8 \mathrm{~Hz}, 1 \mathrm{H}) ; \mathrm{C}^{13} \mathrm{NMR}\left(75 \mathrm{MHz}, \mathrm{CDCl}_{3}\right) \delta_{\mathrm{C}}: 16.81,80.5,97.1,116.7,122.5,124.8,125.3$, 131.2, 131.3, 132.6, 134.6, 135.6, 145.9, 162.1 and 194.8. Anal. Calcd. for $\mathrm{C}_{18} \mathrm{H}_{13} \mathrm{FN}_{2} \mathrm{O}_{5} \mathrm{~S}: \mathrm{C}$, 55.67; H, 3.37; N, 7.21\%. Found: C, 55.63; H, 3.36; N, 7.18\%.

\section{1-(4-Bromophenyl)-3a,8b-dihydroxy-2-(methylthio)-3-nitro-1,8b-dihydroindeno[1,2-b]}

\section{pyrrol-4(3aH)-one (5k)}

Isolated as pale yellow solid; Yield 93\%; m.p. $182-184{ }^{\circ} \mathrm{C} ;{ }^{1} \mathrm{H}$ NMR $\left(300 \mathrm{MHz}, \mathrm{CDCl}_{3}\right) \delta_{\mathrm{H}}$ : $2.11(\mathrm{~s}, 3 \mathrm{H}), 4.85(\mathrm{~s}, 1 \mathrm{H}), 5.76(\mathrm{~s}, 1 \mathrm{H}), 6.57(\mathrm{~d}, J=7.5 \mathrm{~Hz}, 1 \mathrm{H}), 7.25-7.27(\mathrm{~m}, 2 \mathrm{H})$, 7.49-7.65 (m, 4H), 7.87-7.91 (m, 1H), $\mathrm{C}^{13} \mathrm{NMR}\left(75 \mathrm{MHz}, \mathrm{CDCl}_{3}\right) \delta_{\mathrm{C}}: 16.9,80.6,97.3$, 122.8, 123.4, 124.8, 125.2, 130.7, 131.2, 132.9, 134.5, 135.7, 145.7 and 194.7. Anal. Calcd. for $\mathrm{C}_{18} \mathrm{H}_{13} \mathrm{BrN}_{2} \mathrm{O}_{5} \mathrm{~S}$ : C, 48.12; H, 2.92; N, 6.24\%. Found: C, 48.07; H, 2.88; N, 6.21\%. 


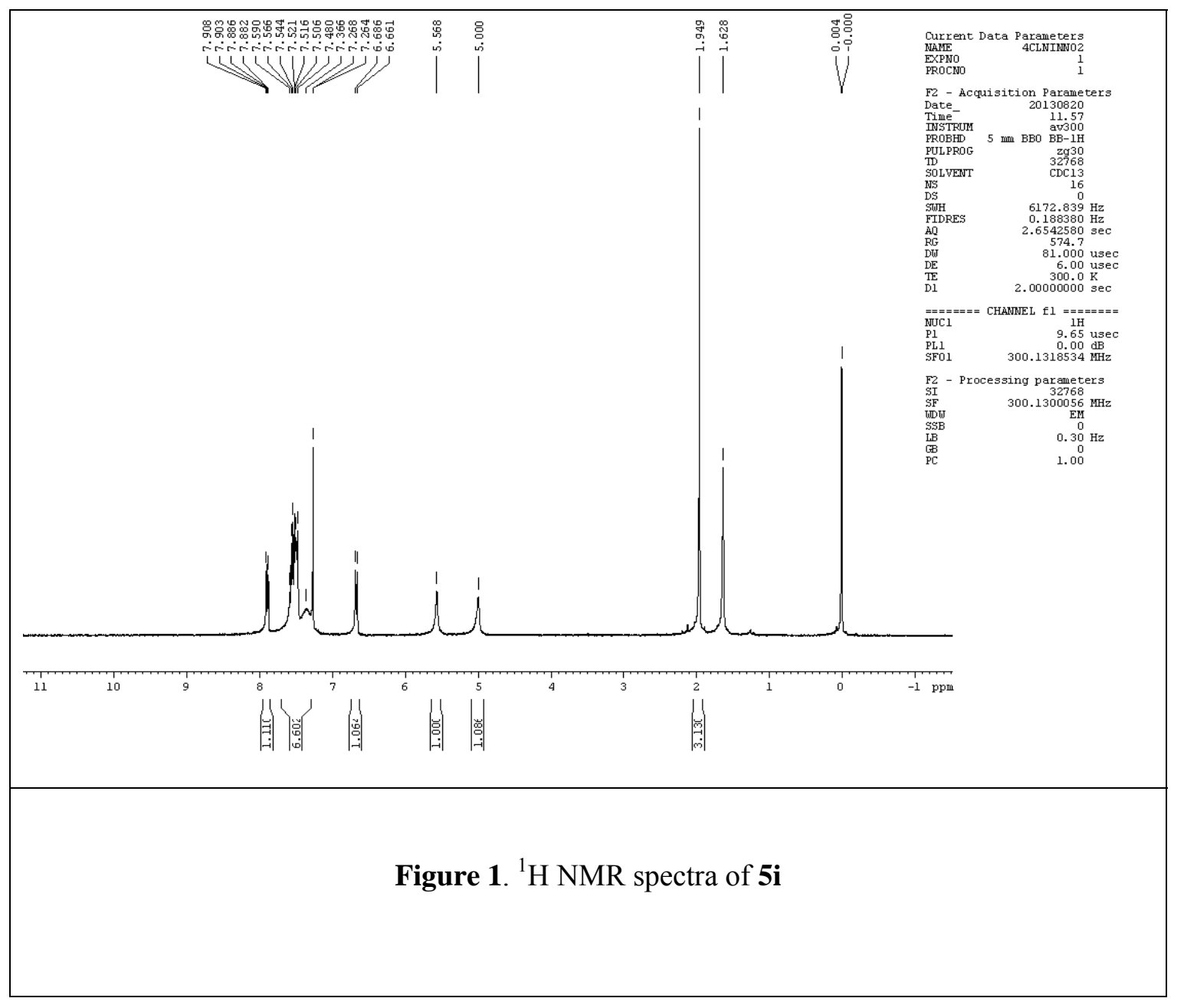




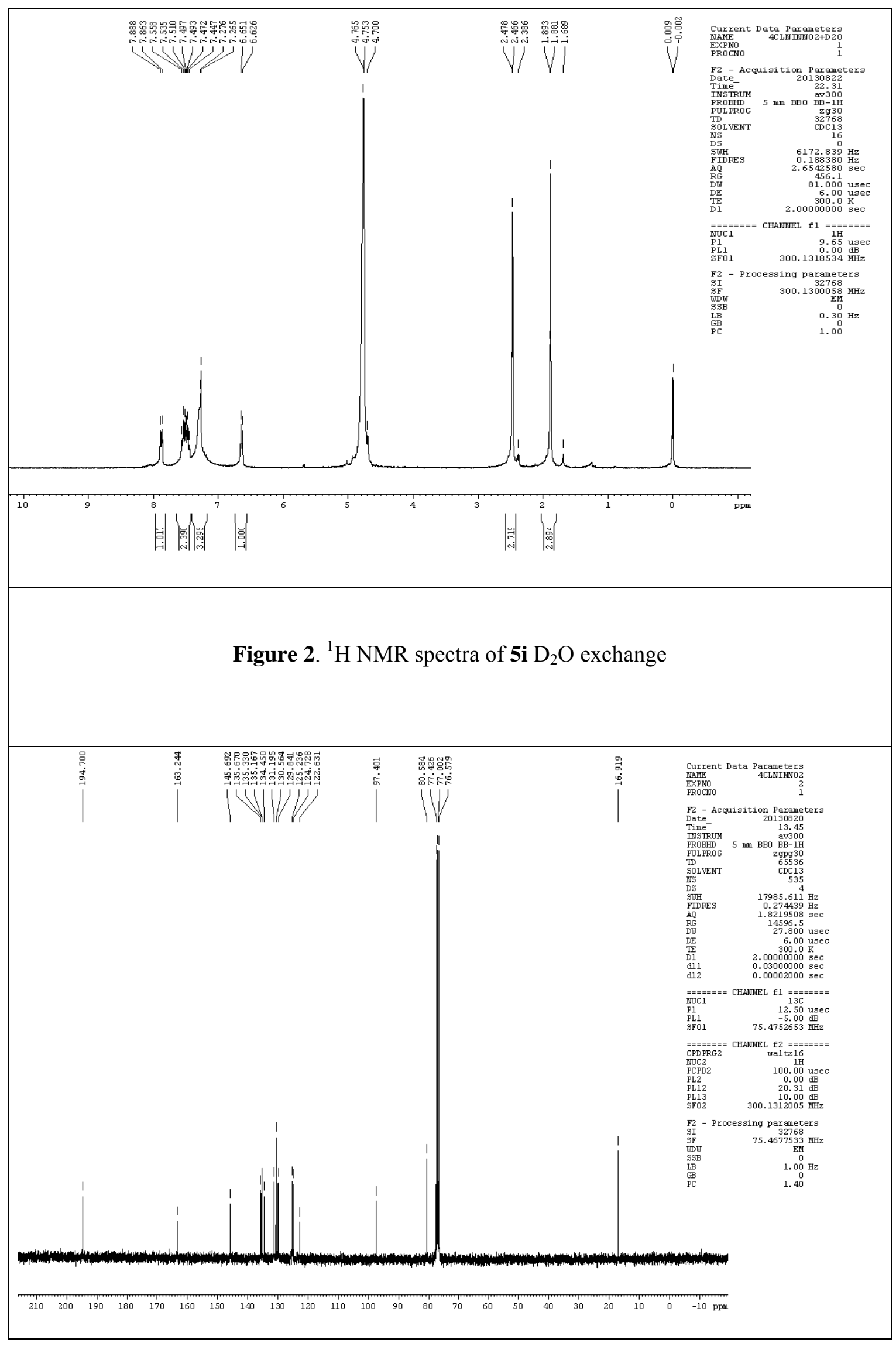




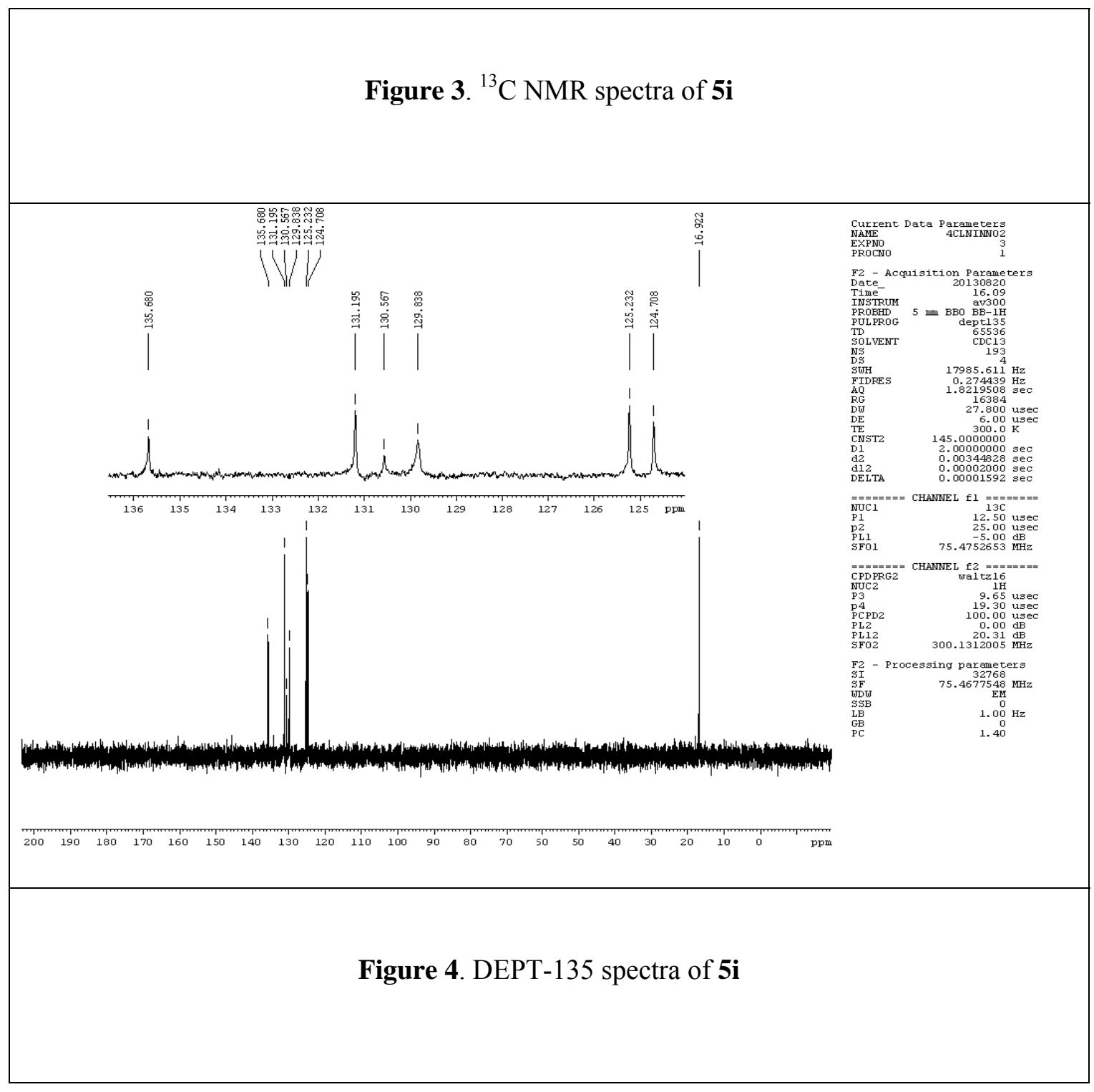




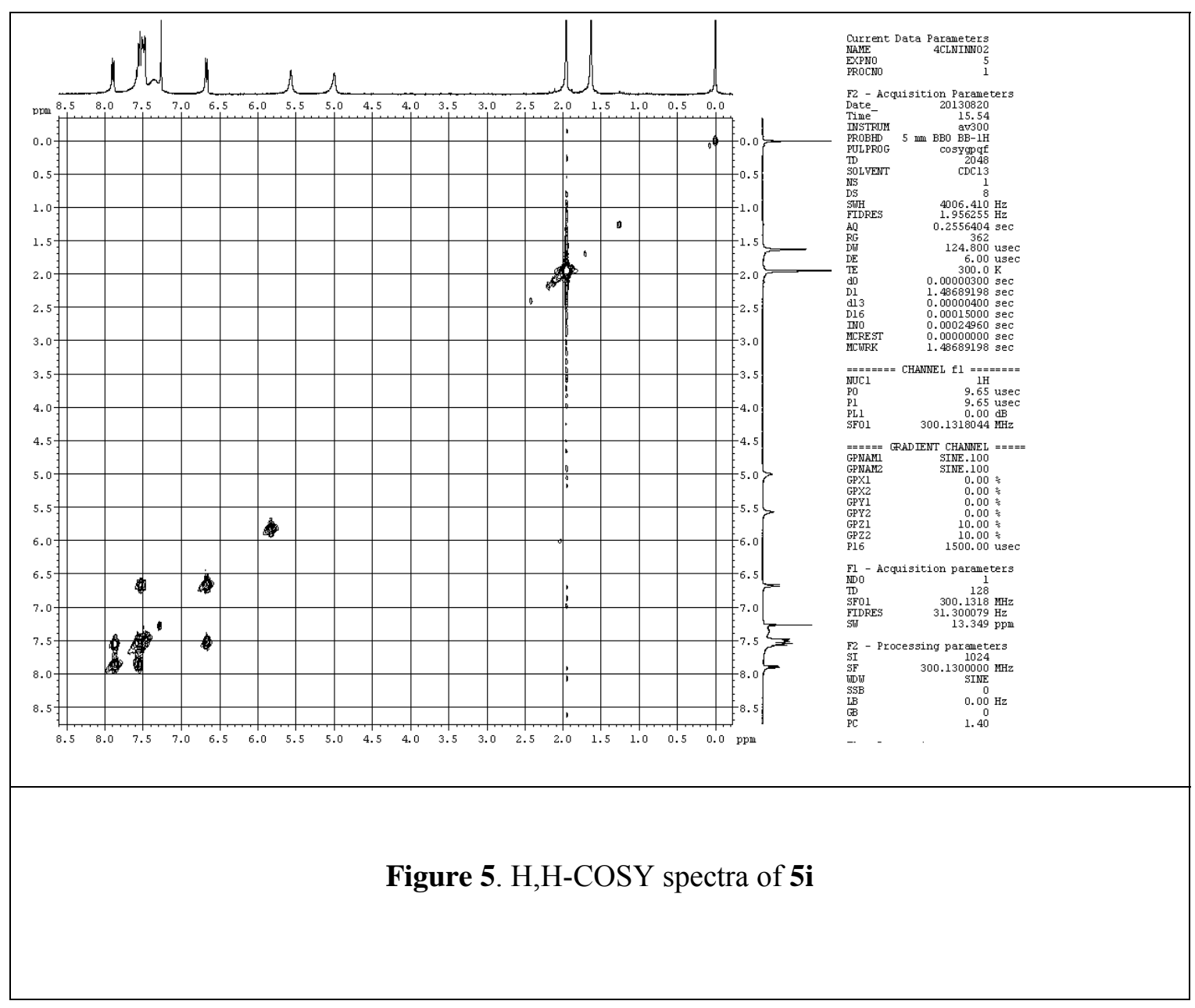




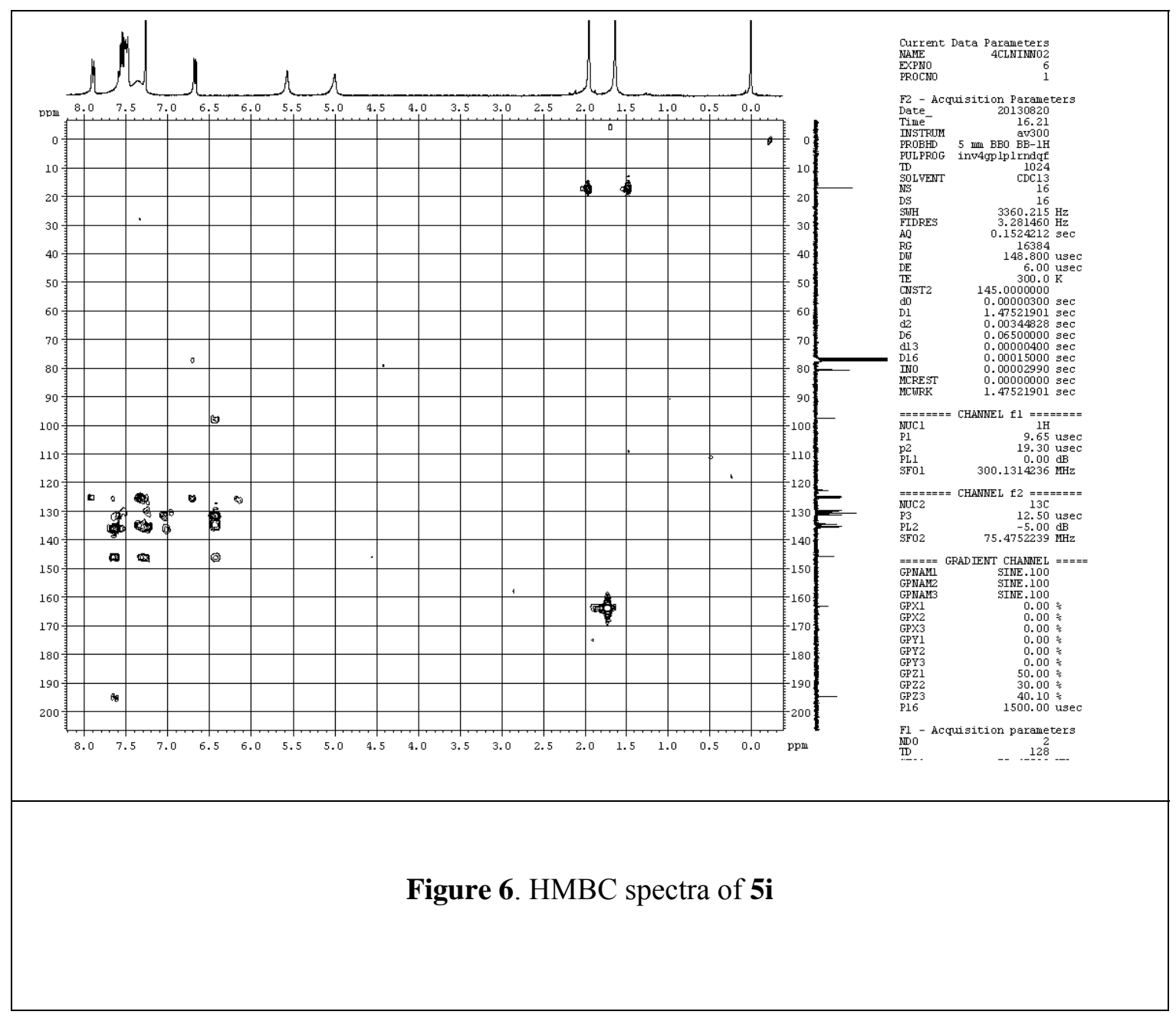




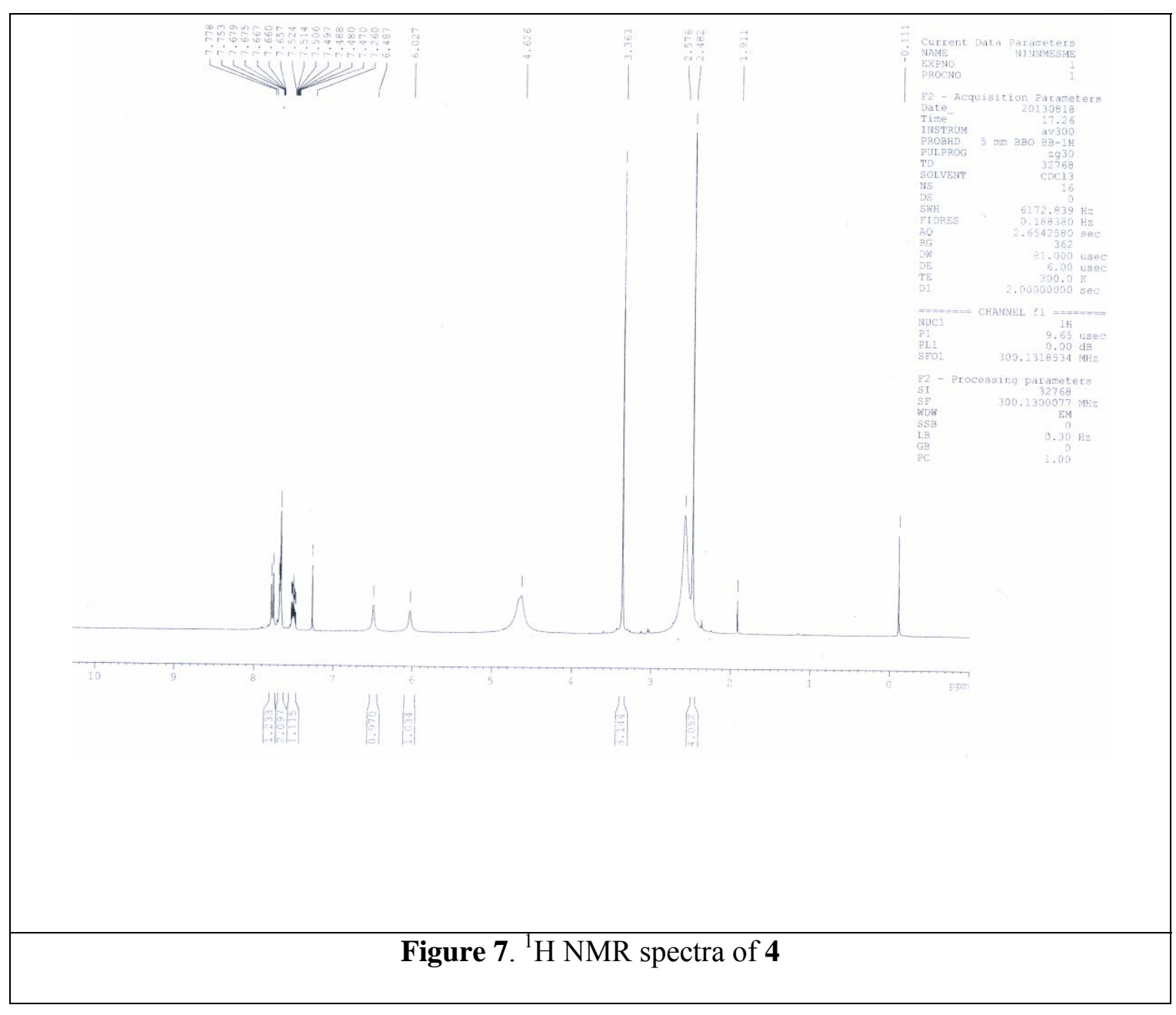




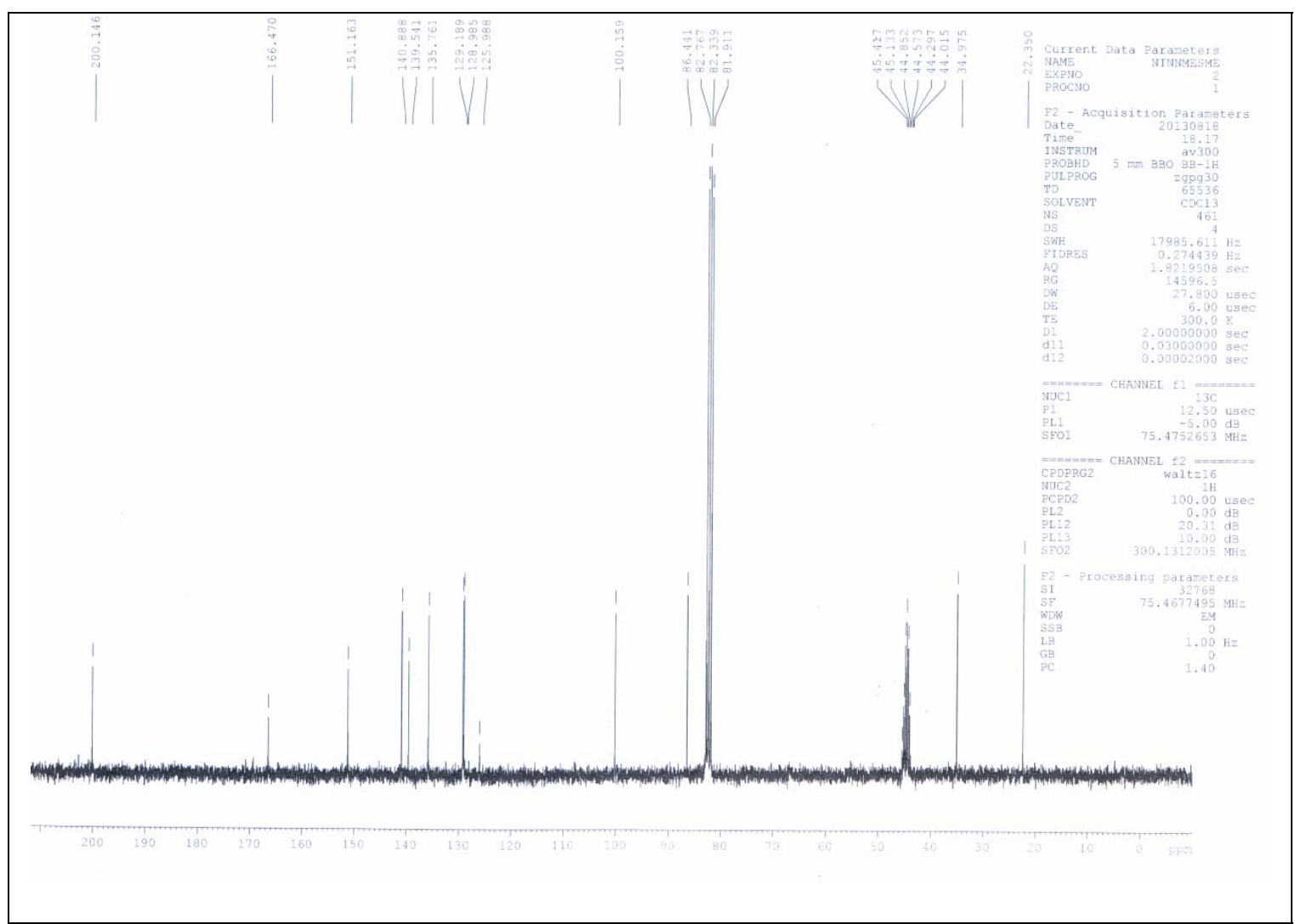

Figure 8. ${ }^{13} \mathrm{C}$ NMR spectra of 4 


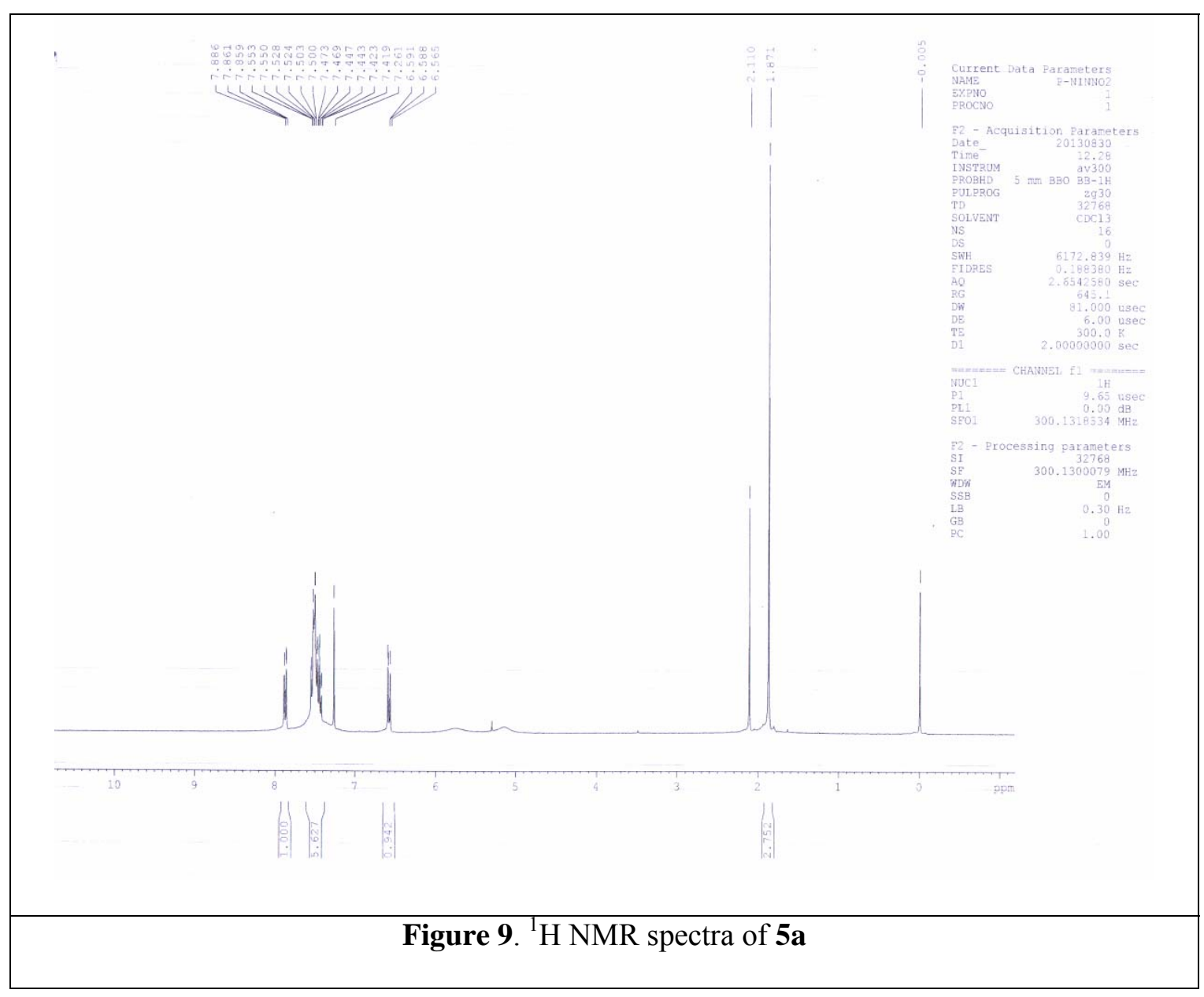




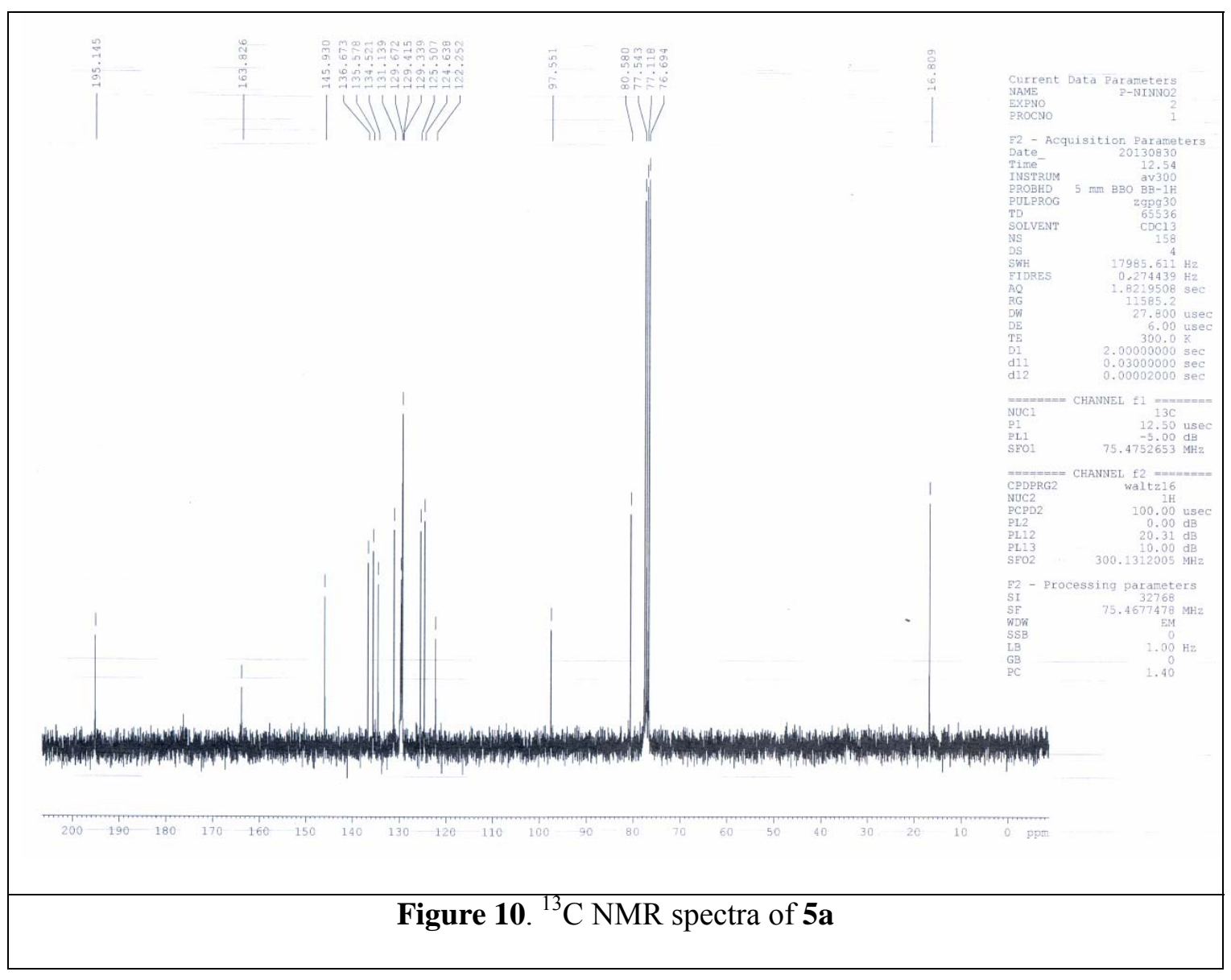




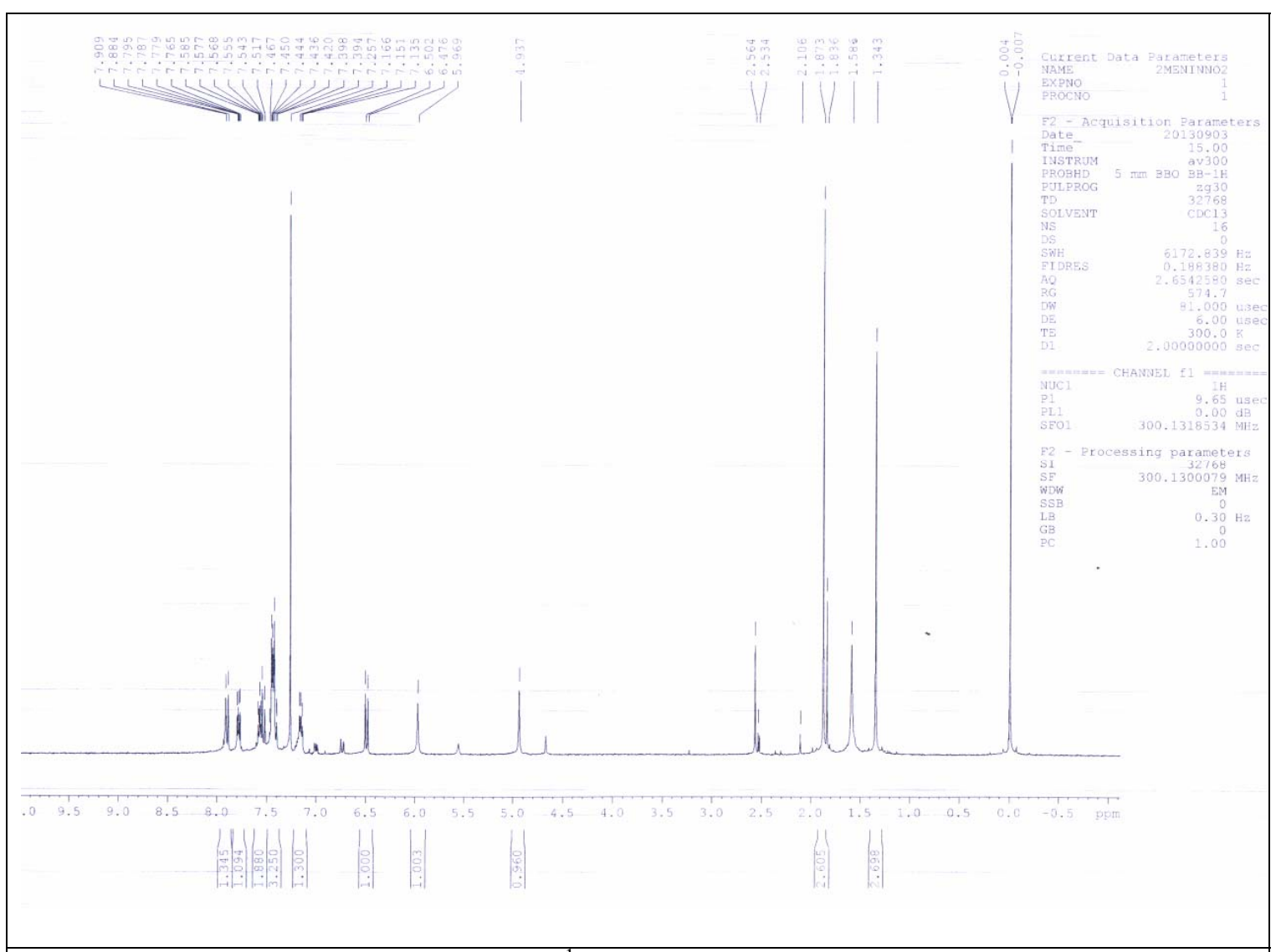

Figure 11. ${ }^{1} \mathrm{H}$ NMR spectra of $\mathbf{5 b}$ 


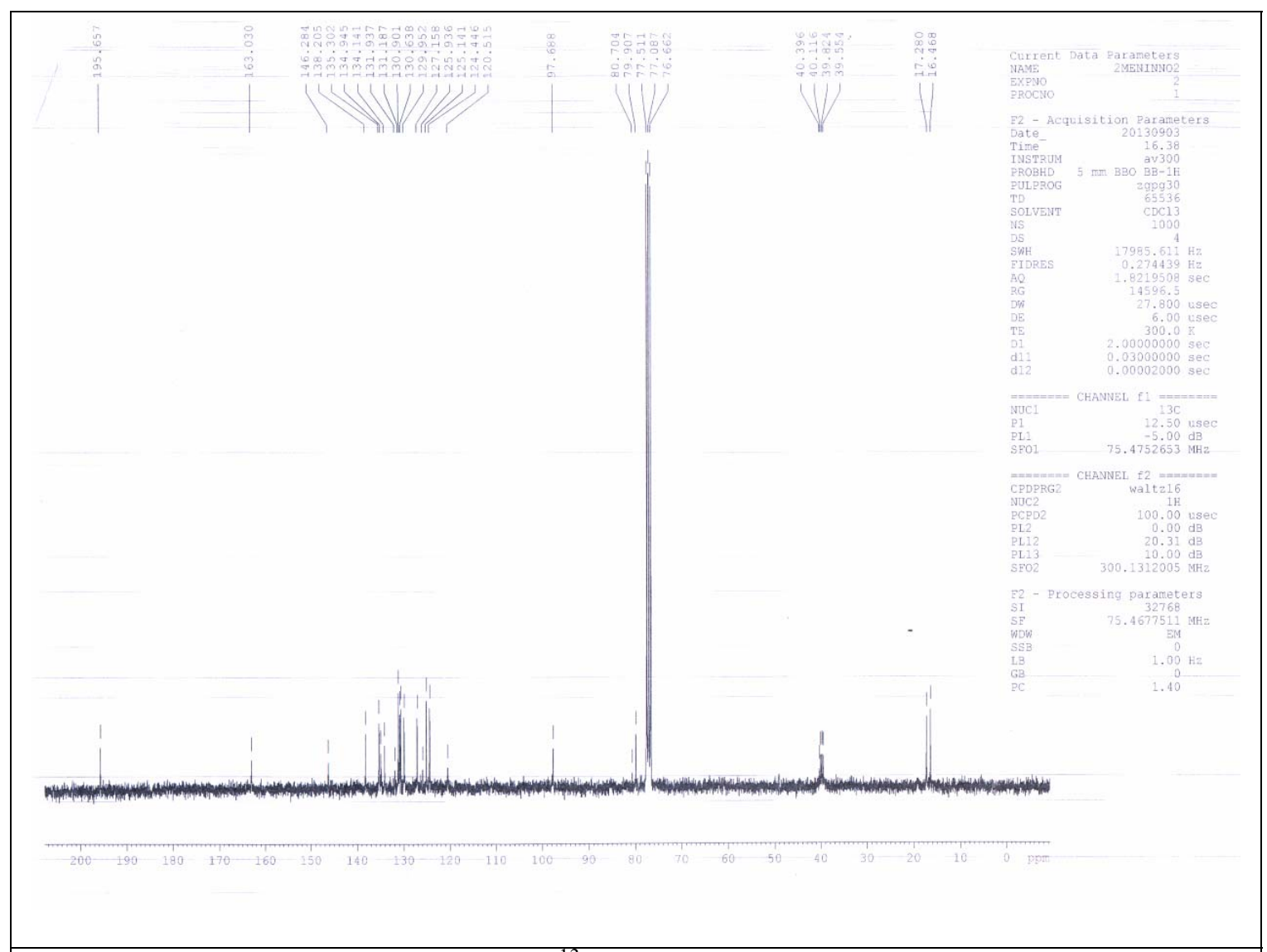

Figure 12. ${ }^{13} \mathrm{C}$ NMR spectra of $\mathbf{5 b}$ 


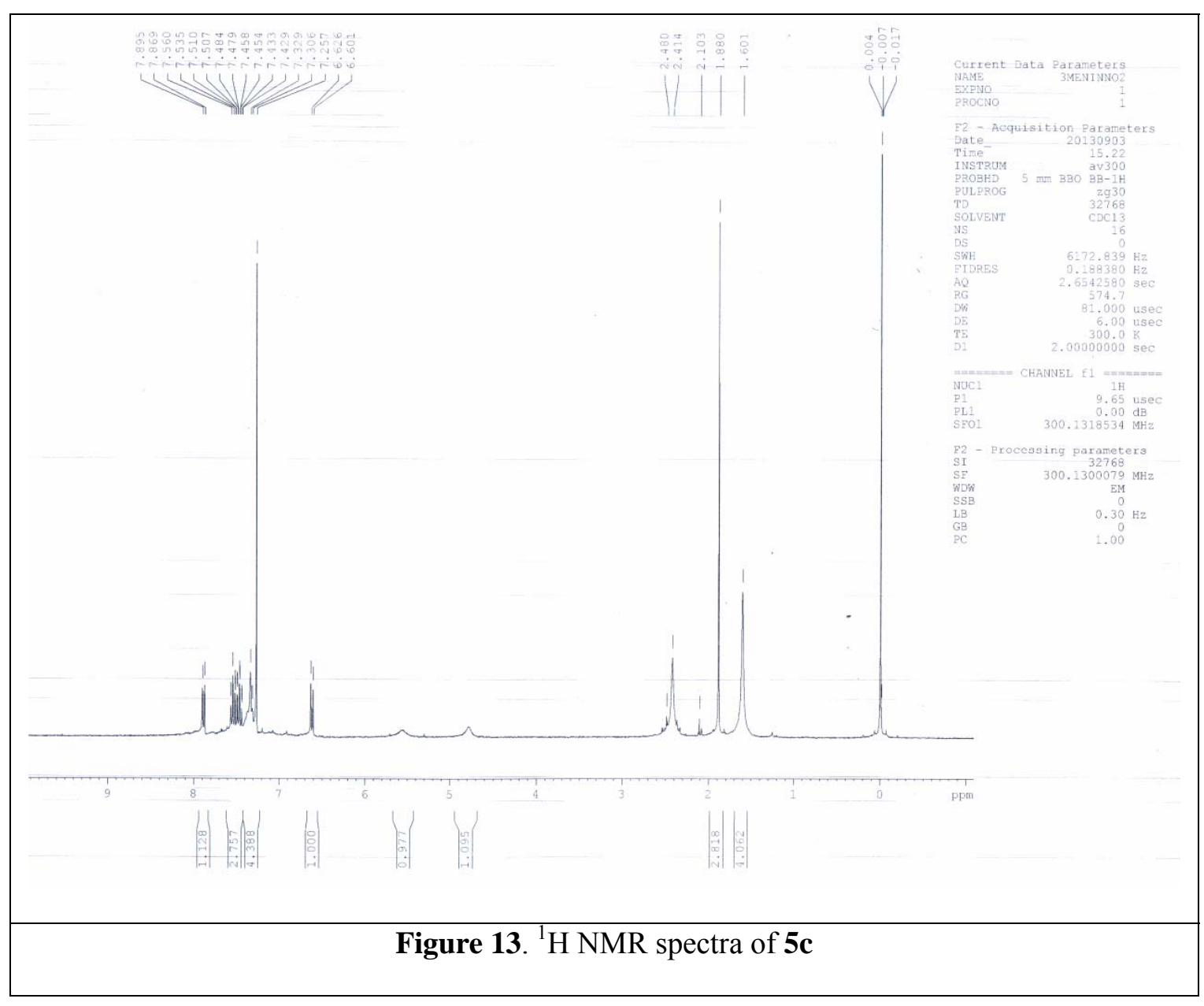




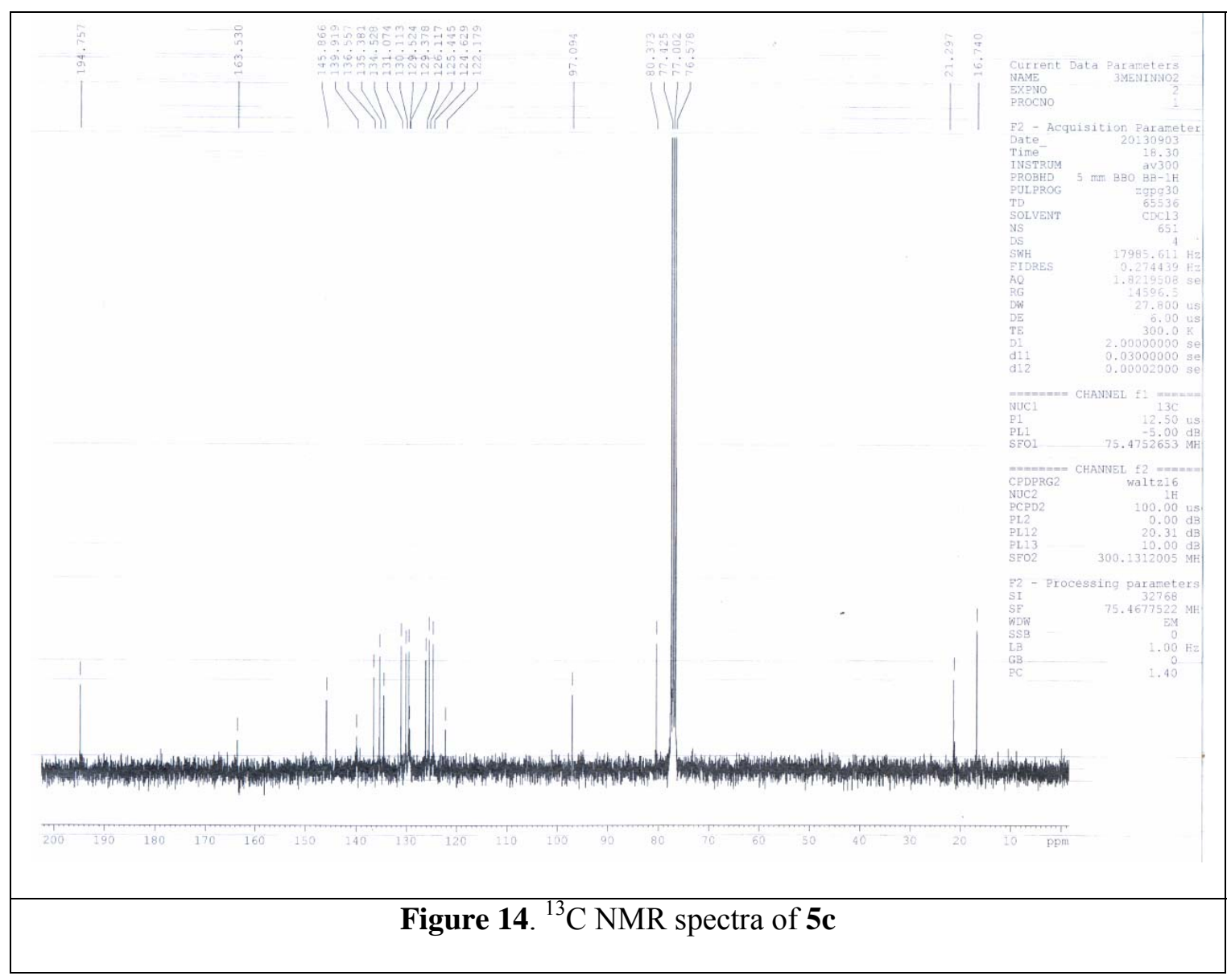




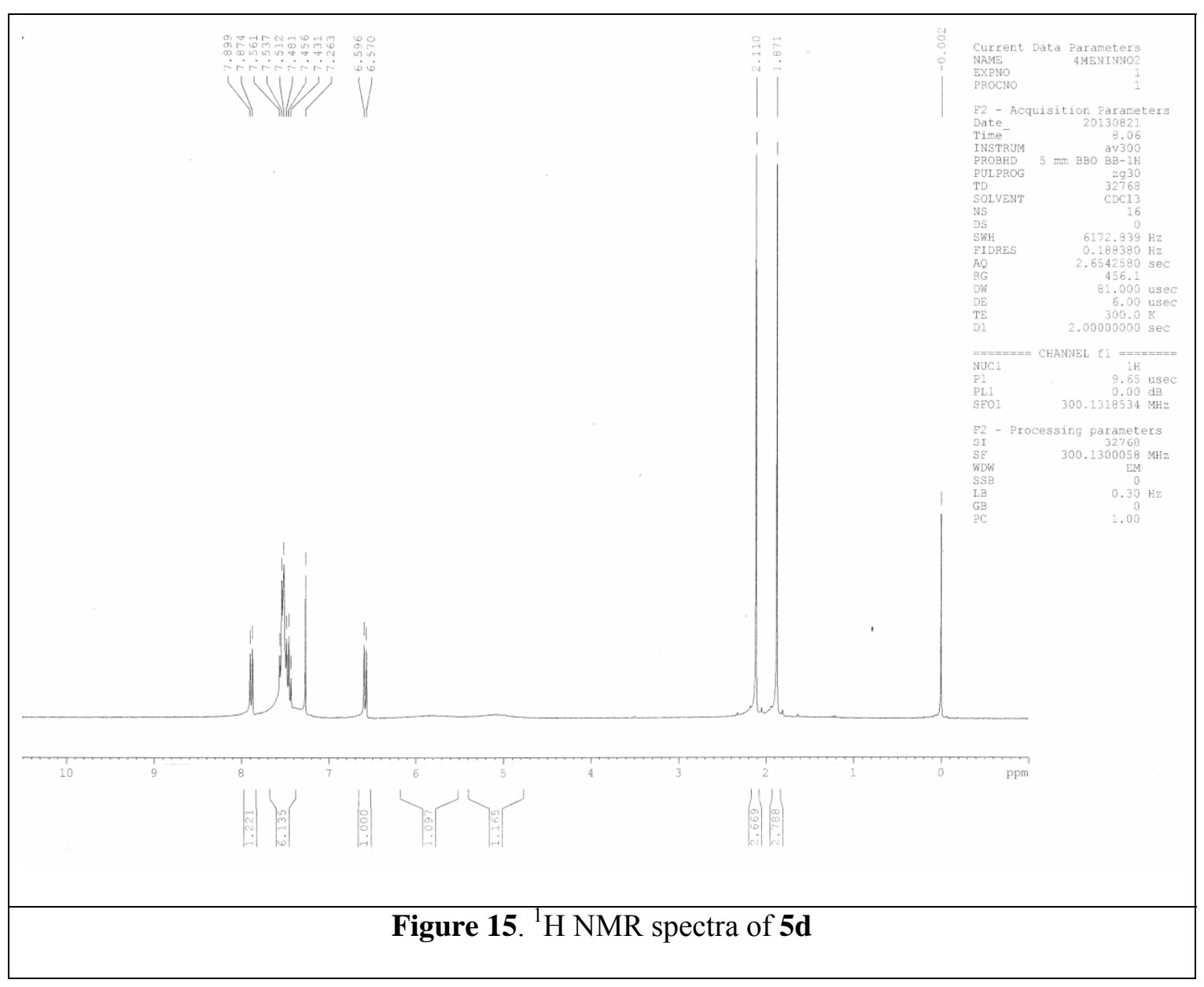




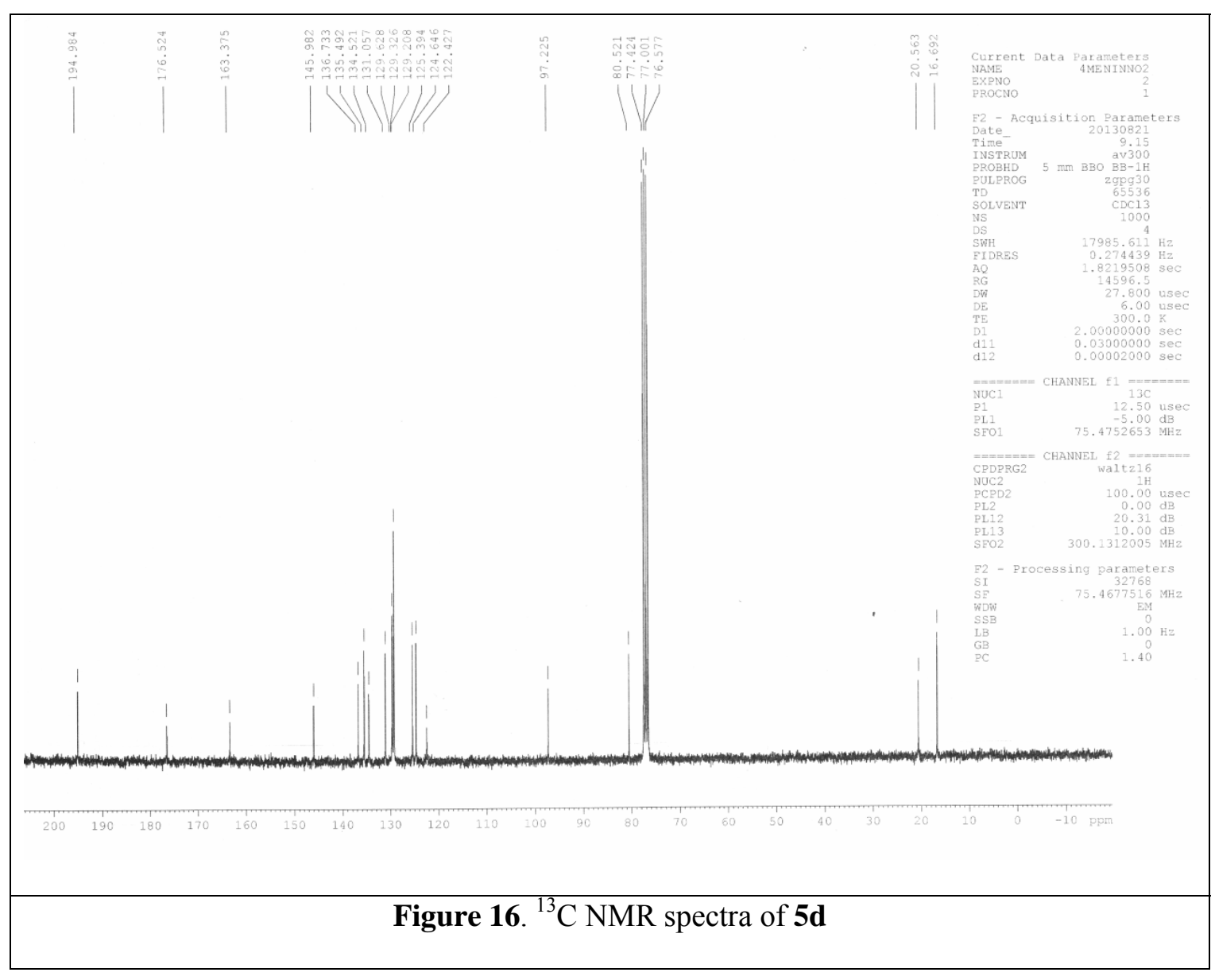




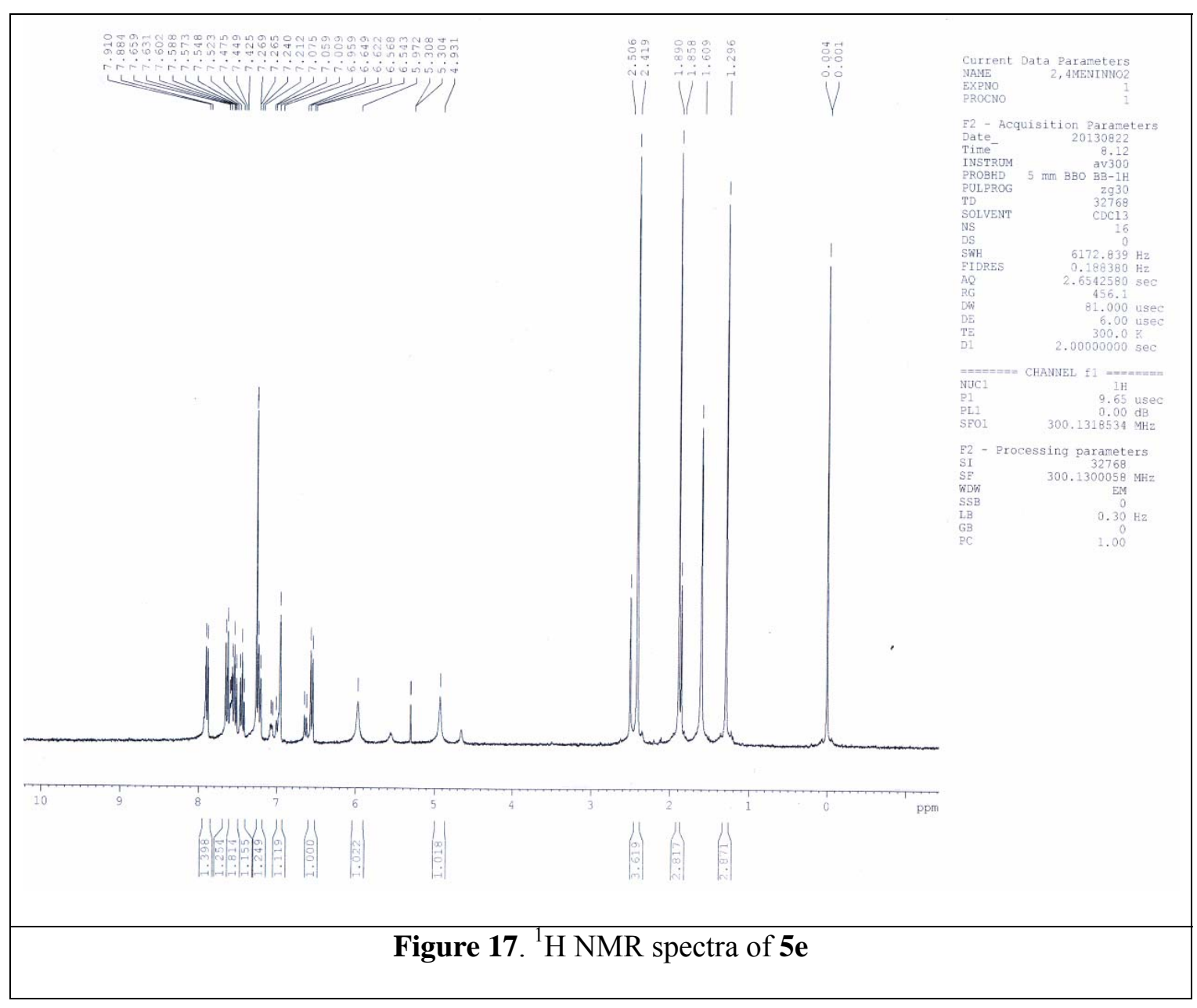




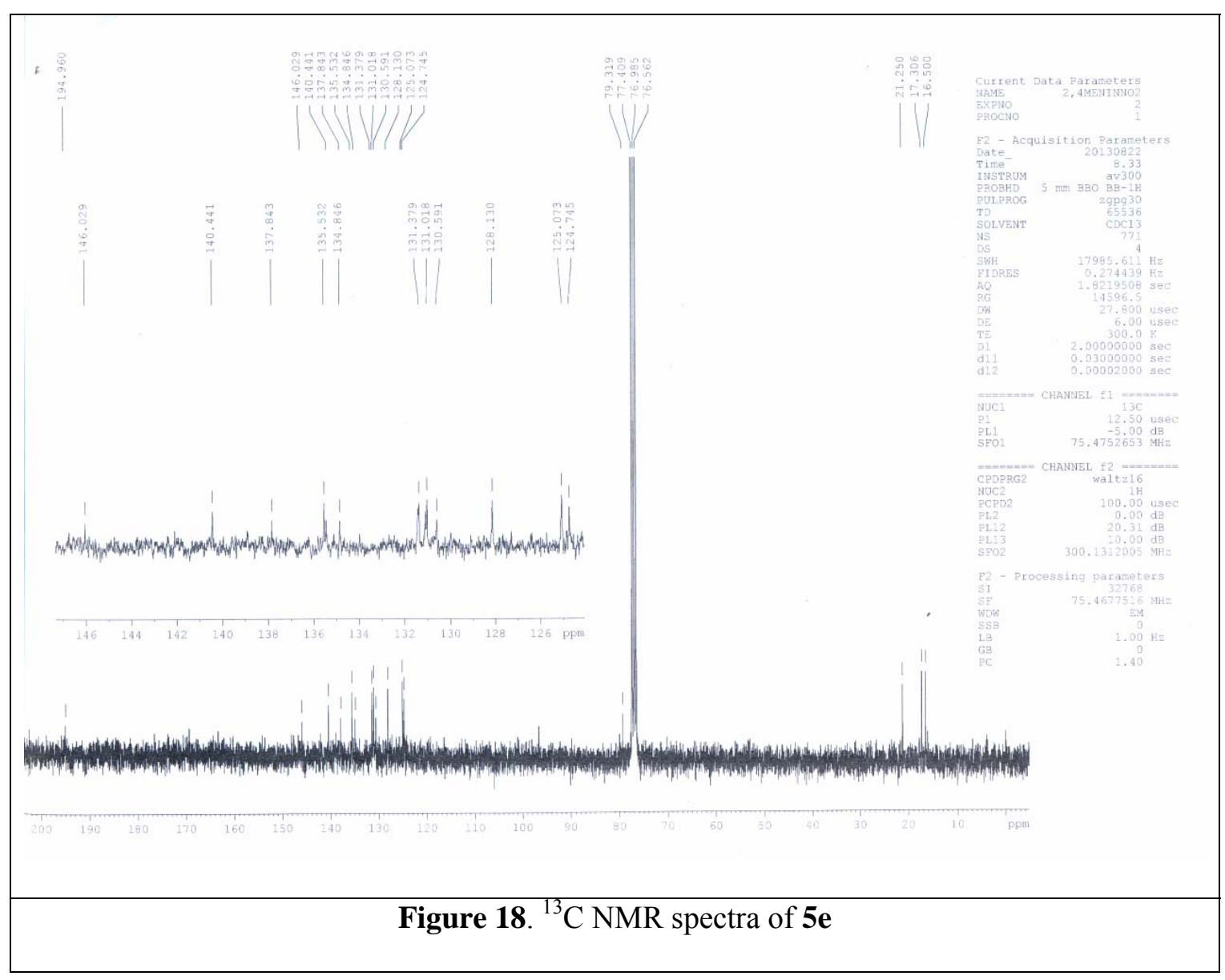




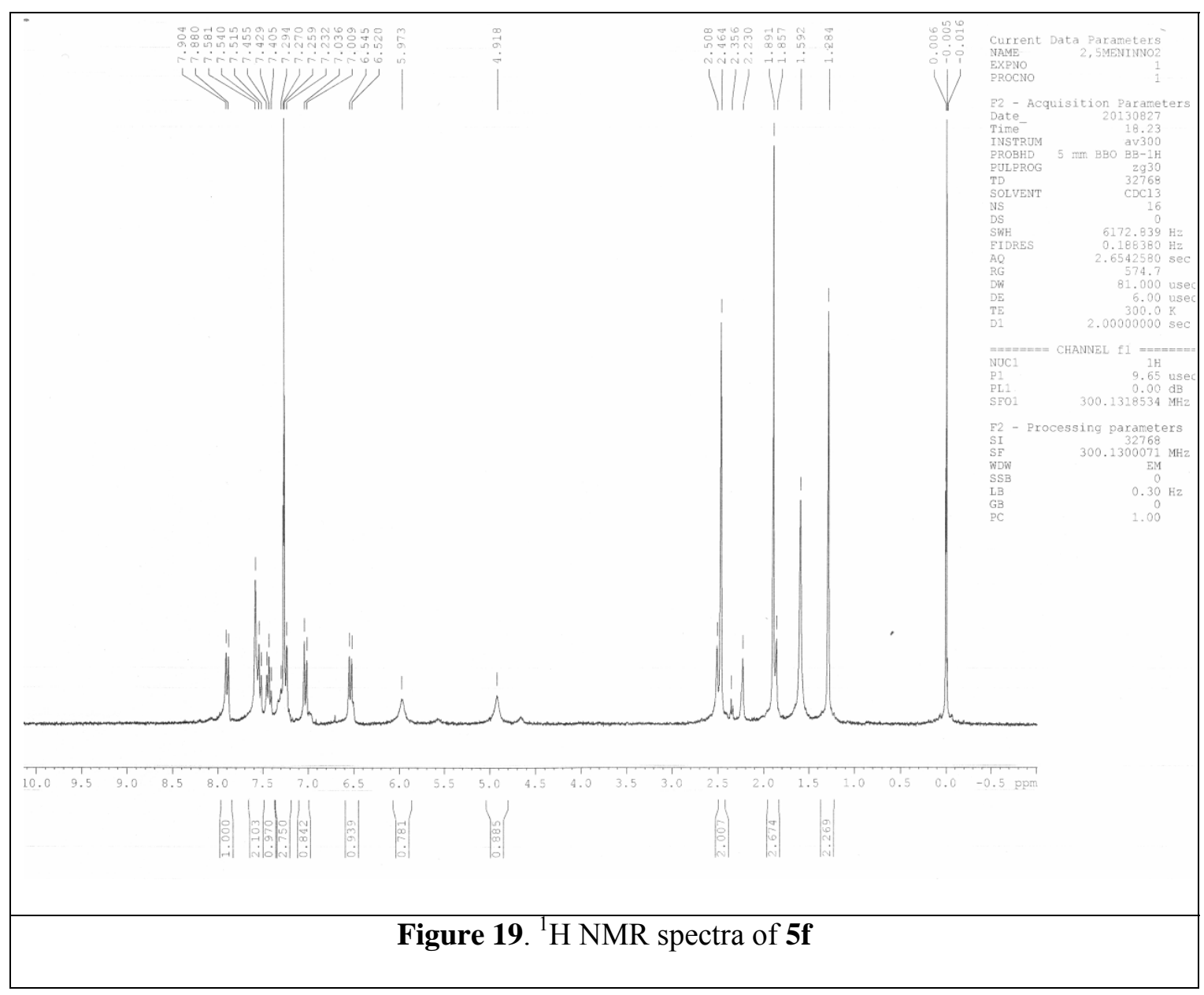




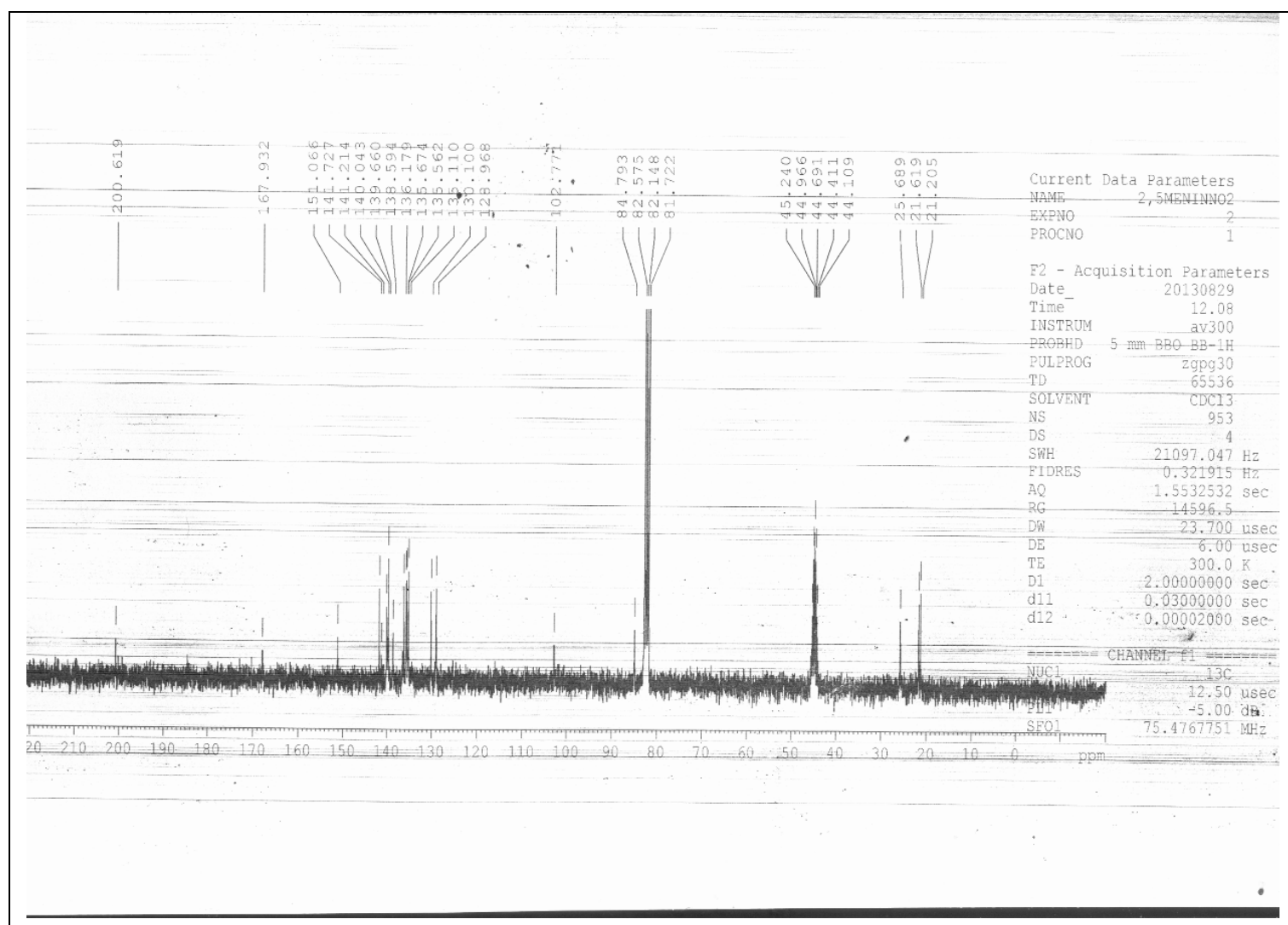

Figure 20. ${ }^{13} \mathrm{C}$ NMR spectra of $\mathbf{5 f}$ 


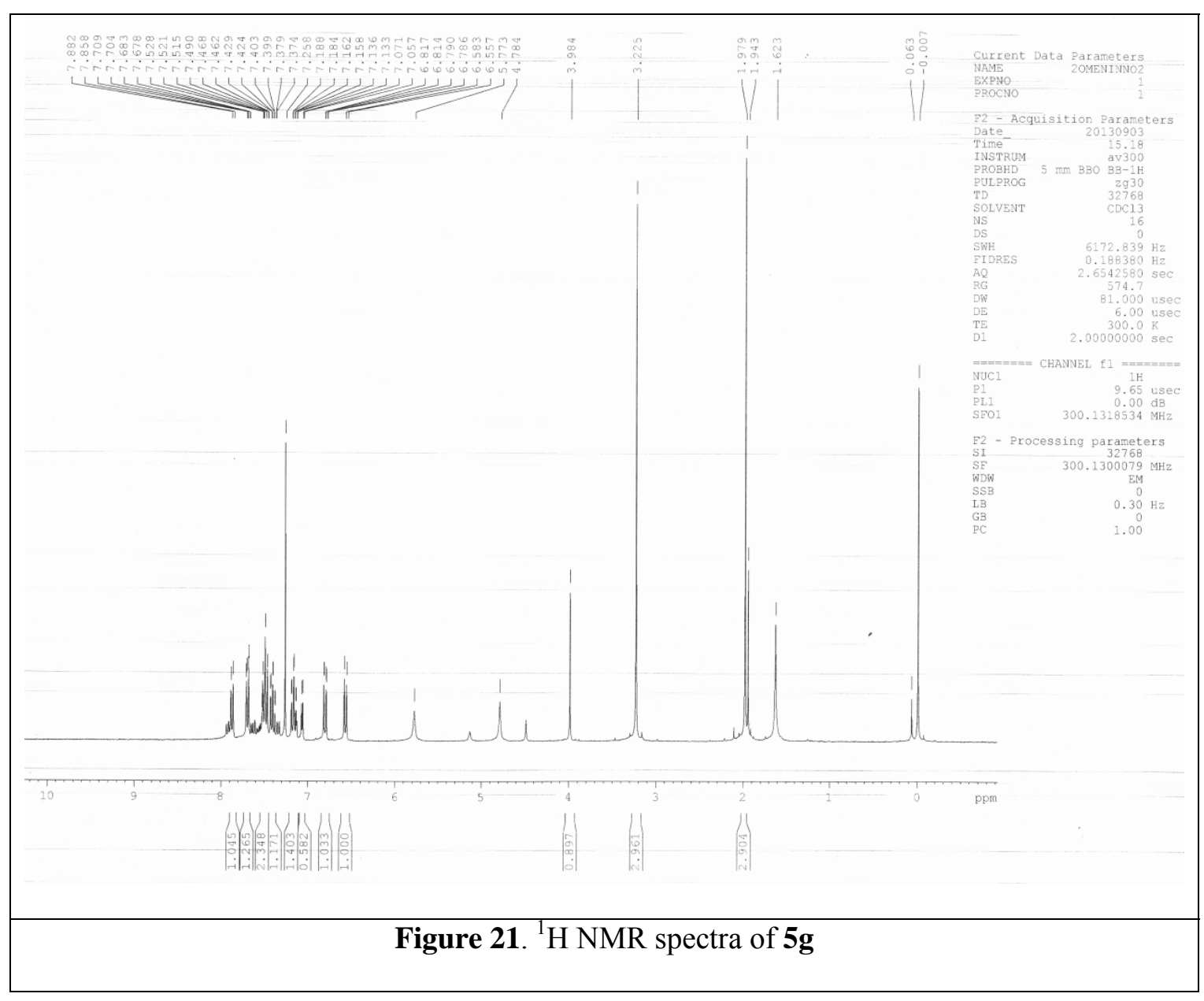




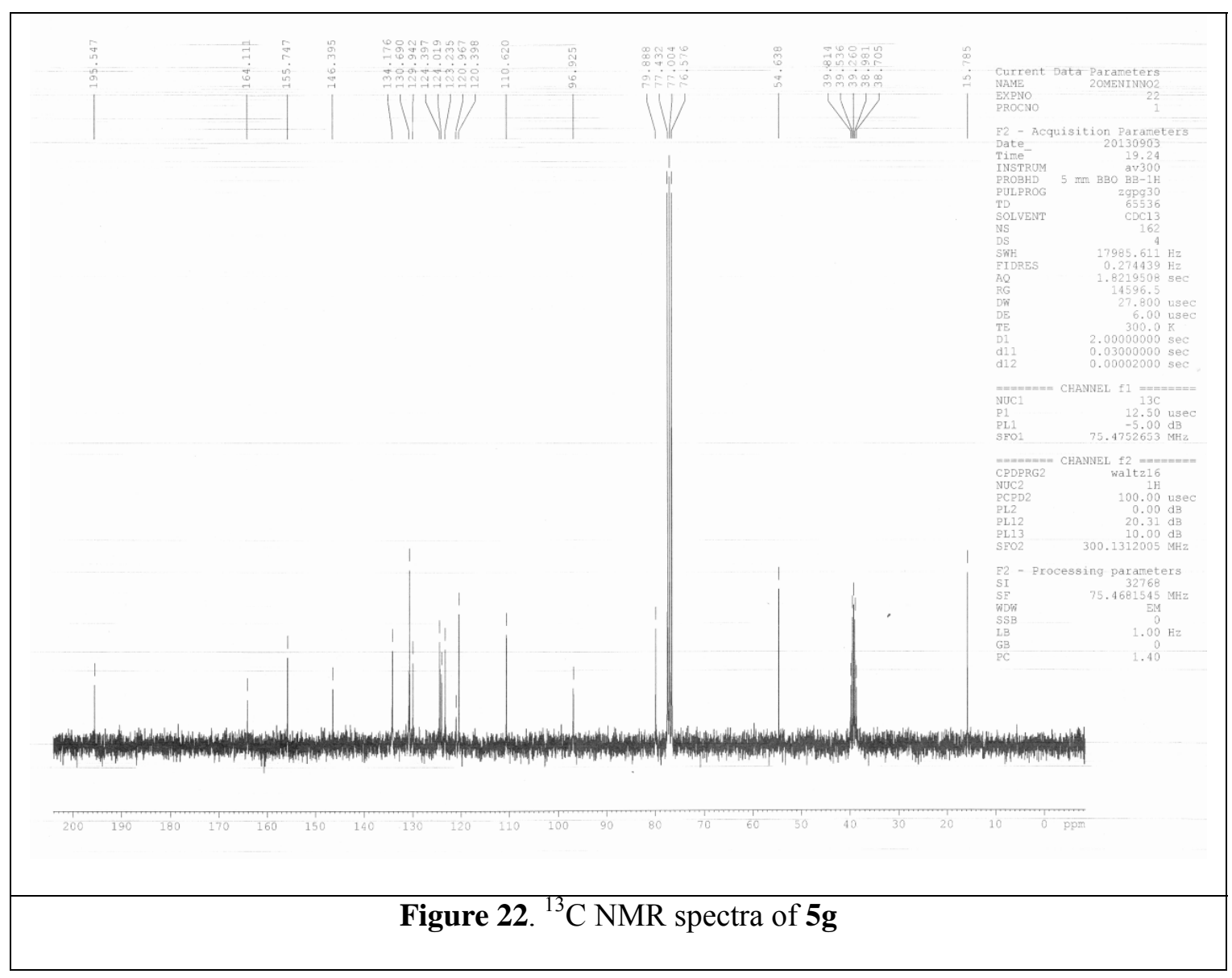




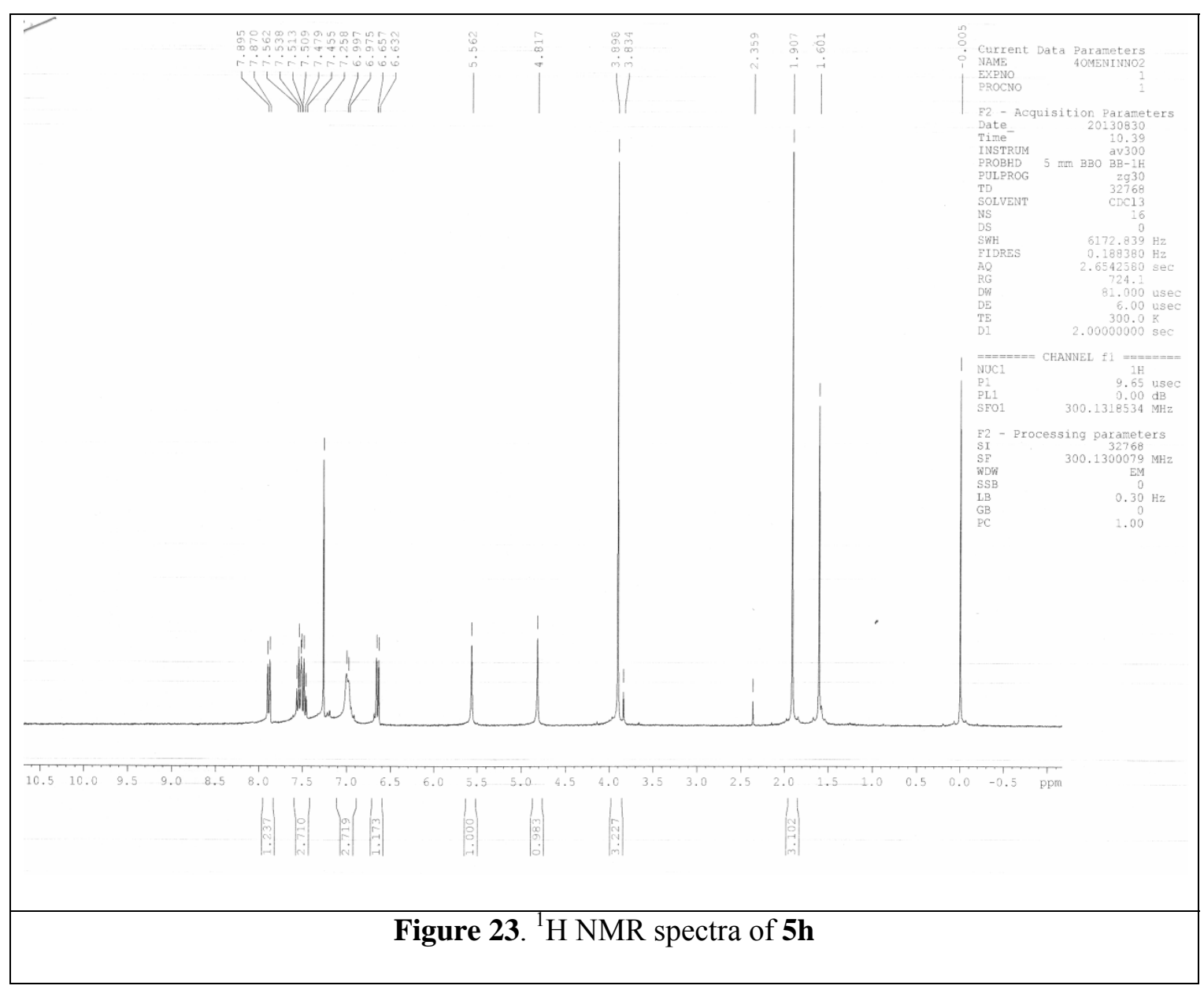




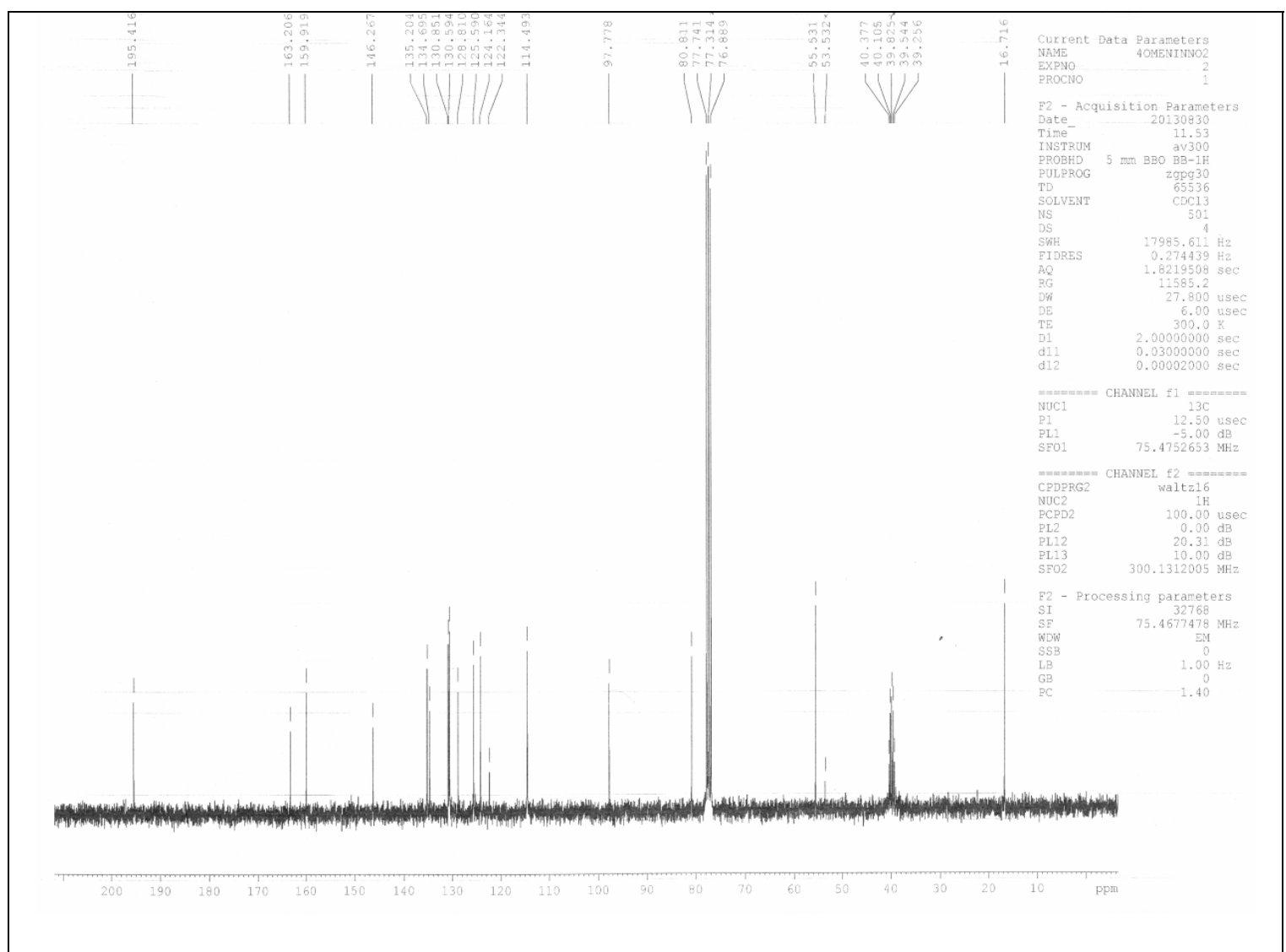

Figure 24. ${ }^{13} \mathrm{C}$ NMR spectra of $\mathbf{5 h}$ 


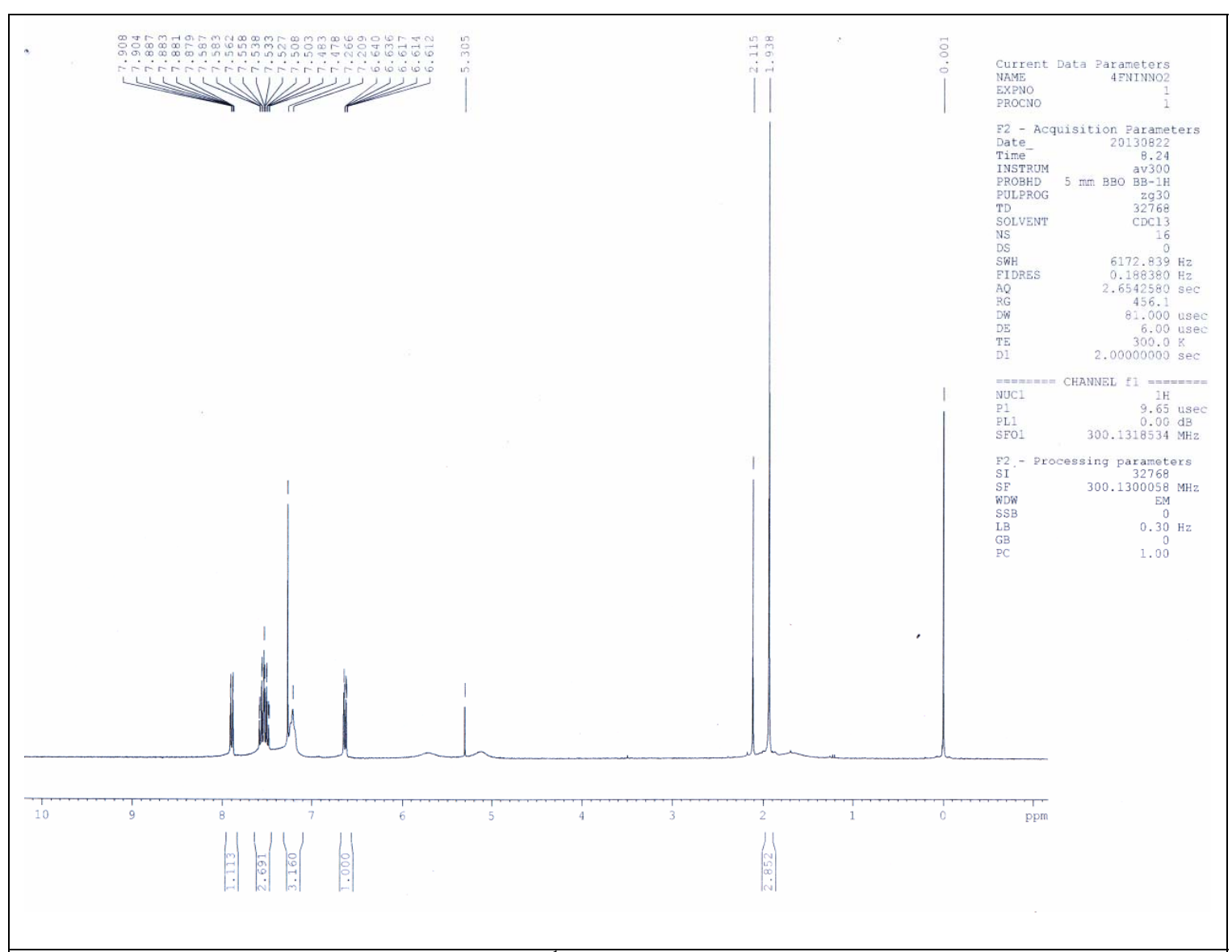

Figure 25. ${ }^{1} \mathrm{H}$ NMR spectra of $\mathbf{5 j}$ 


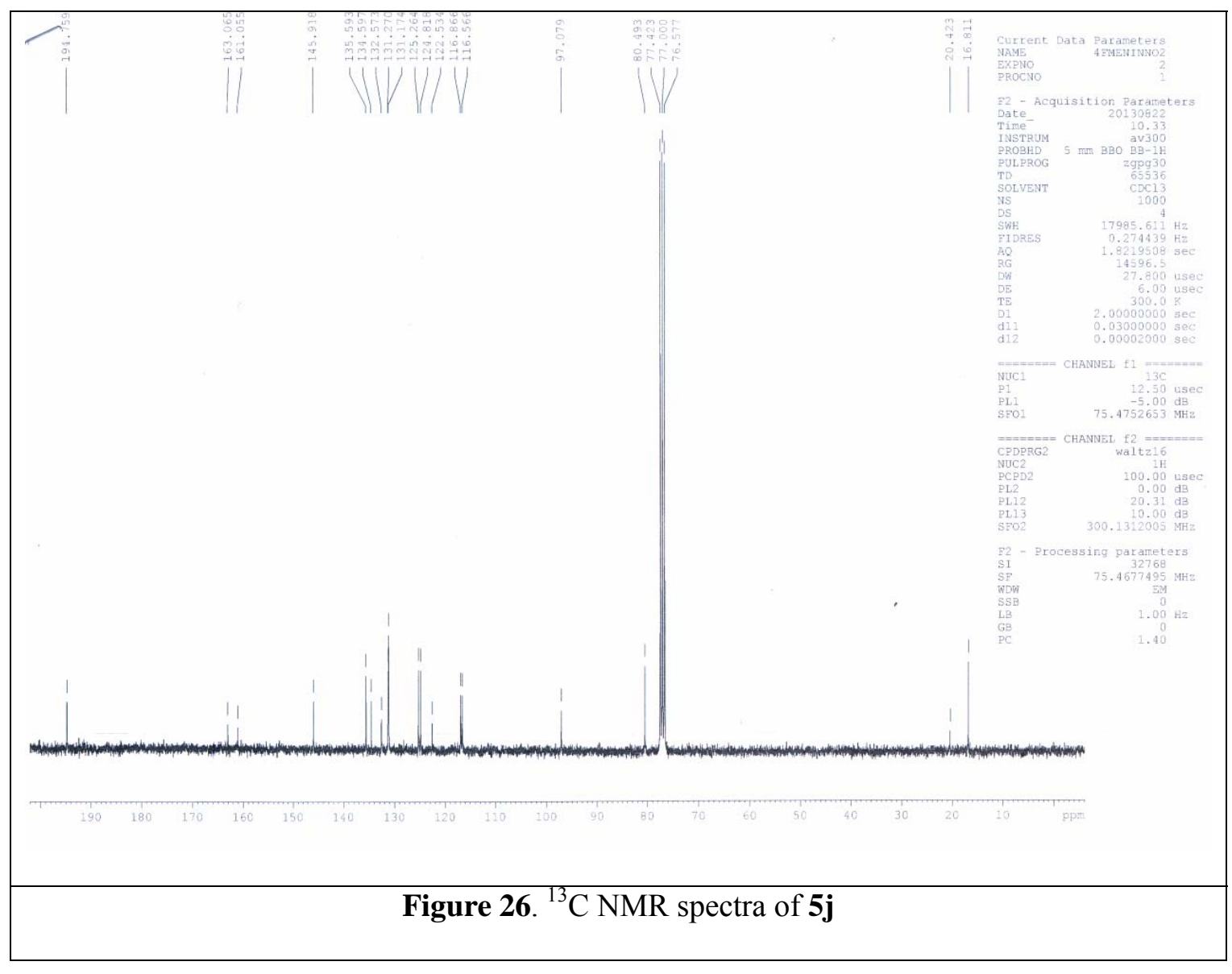




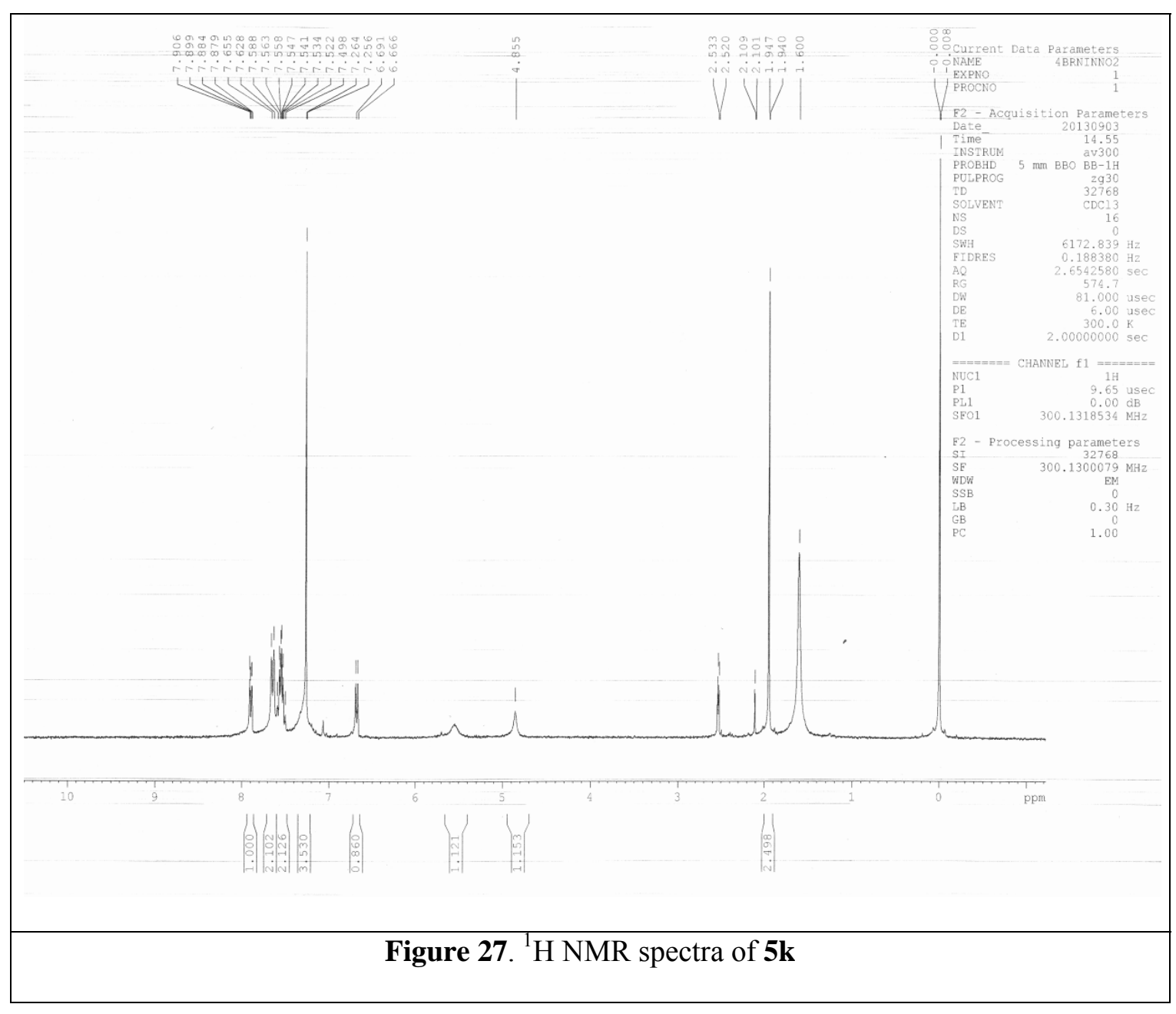




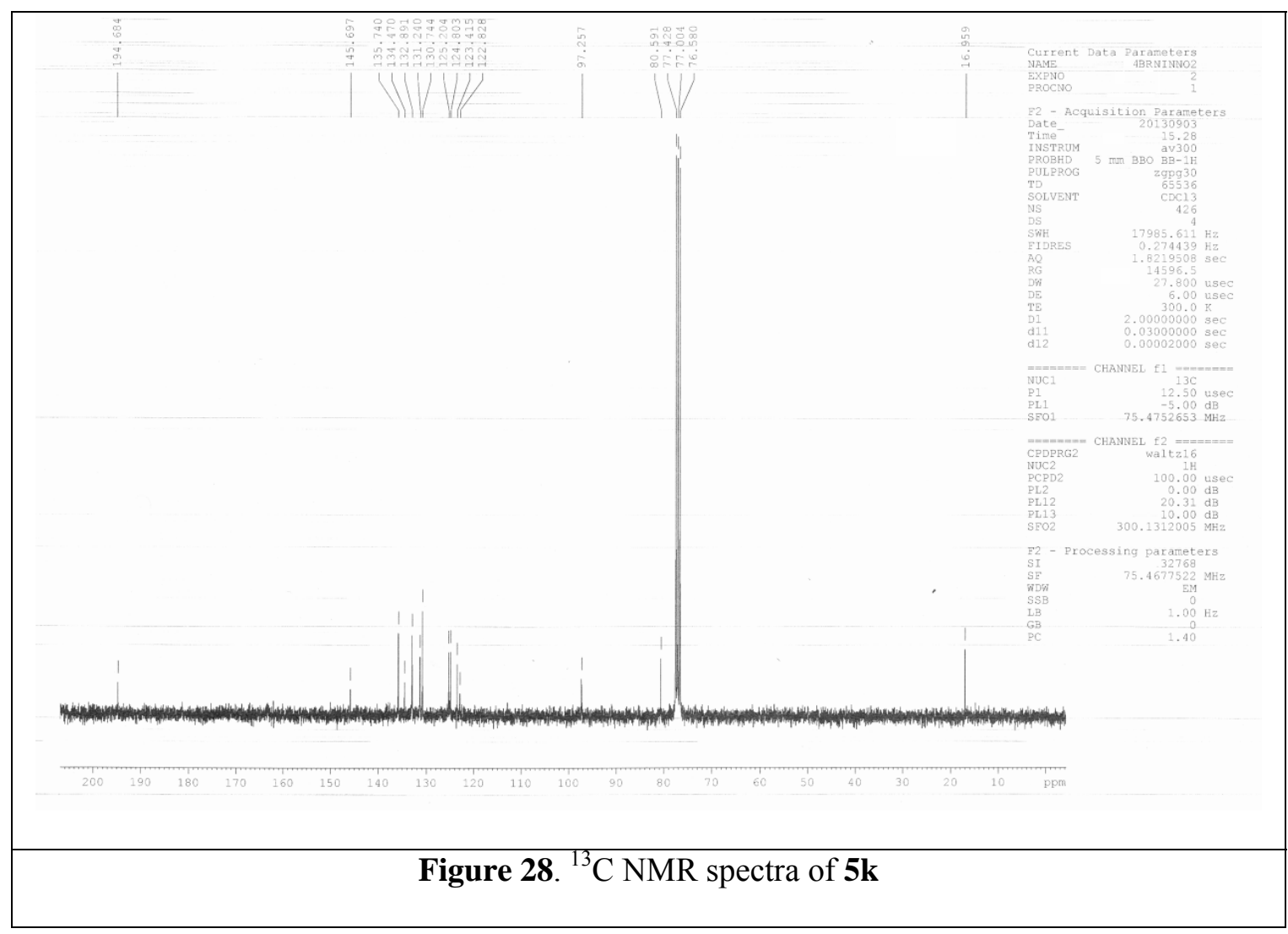

